\title{
OPTIMAL INVESTMENT AND CONSUMPTION WITH LABOR INCOME IN INCOMPLETE MARKETS
}

\author{
OLEKSII MOSTOVYI AND MIHAI SÎRBU
}

\begin{abstract}
We consider the problem of optimal consumption from labor income and investment in a general incomplete semimartingale market. The economic agent cannot borrow against future income, so the total wealth is required to be positive at (all or some) previous times. Under very general conditions, we show that an optimal consumption and investment plan exists and is unique, and provide a dual characterization in terms of an optional strong supermartingale deflator and a decreasing part, which charges only the times when the no-borrowing constraint is binding. The analysis relies on the infinite-dimensional parametrization of the income/liability streams and, therefore, provides the first-order dependence of the optimal investment and consumption plans on future income/liabilities (as well as a pricing rule). An emphasis is placed on mathematical generality.
\end{abstract}

\section{INTRODUCTION}

Optimal investment with intermediate consumption and a stream of labor income (or liabilities) is one of the central problems in mathematical economics. If borrowing against the future income is prohibited, the main technical difficulty lies in the fact that there are infinitely many constraints. Even in the deterministic case of no stocks and non-random income, a classical approach is based on the convexification of the constraints that leads to a non-trivial dual problem formulated over decreasing nonnegative functions.

Borrowing constraints imposed at all times not only affect the notion of admissibility, leading to more difficult mathematical analysis, but also change the meaning to fundamental concepts of mathematical finance such as replicability and completeness. The latter

Date: January 29, 2019.

2010 Mathematics Subject Classification. 91G10, 93E20. JEL Classification: C61, G11.

Key words and phrases. Utility maximization, optimal investment, unified framework of admissibility, labor income, local martingale measure, duality theory, semimartingale, incomplete market, random endowment, admissibility, complementary slackness, optional strong supermartingale, optional strong supermartingale deflator, Skorokhod's representation theorem.

The first author has been supported by the National Science Foundation under grant No. DMS1600307 (2015 - 2018), the second author supported by the National Science Foundation under grant No. DMS-1517664 (2015 - 2018). Any opinions, findings, and conclusions or recommendations expressed in this material are those of the authors and do not necessarily reflect the views of the National Science Foundation. 
is formulated via the attainability of every (bounded) contingent claim by a portfolio of traded assets. For a labor income/liability streams that pays off dynamically, there is no a priori guarantee that such a replicating portfolio (if it exists at all) is admissible, i.e., satisfies the constraints. Thus, in the terminology of [HP93], even a complete market becomes dynamically incomplete under the borrowing constraints. The analysis of such a problem (in otherwise complete Brownian settings with a corresponding unique risk-neutral measure), is performed in [HP93] and later in [EKJP98]. The nonnegative decreasing processes (that parametrize the dynamic incompleteness mentioned above) play an important role in the characterizations of optimal investment and consumption plans. The analysis in [HP93] and [EKJP98] is connected with optimal stopping techniques from [Kar89].

Incomplete markets with no-borrowing constraints have been analyzed only in specific Markovian models in [DZ93] and [DFSZ97] based on partial differential equations techniques. The goal of the present paper is to study the problem of consumption and investment with no-borrowing constraints in general (so, non-Markovian) incomplete models. This leads to having, simultaneously, two layers of incompleteness. One comes from the many martingale measures, the other from a similar class of non-decreasing processes (as above) that describe the dynamic incompleteness. We refer to [MT07] for the examples of market incompleteness in finance and macroeconomics.

In contrast to [HP93] and [EKJP98, our model not only allows for incompleteness, but also for jumps. Mathematically, this means we choose to work in a general semimartingale framework. As in [HP93] and [EKJP98], our approach is based on duality. One of the principal difficulties is in the construction of the dual feasible set and the dual value function. It is well-known that the martingale measures drive the dual domain in many problems of mathematical finance. On the other hand, the convexification of constraints leads to the decreasing processes as the central dual object as well. We show that the dual elements in the incomplete case can be approximated by products of the densities of martingale measures and such nonnegative decreasing processes. This is one of the primary results of this work, see section 5 , that leads to the complementary slackness characterization of optimal wealth in section 6, where it is shown that the approximating sequence for the dual minimizer leads to a nonincreasing process, which decreases at most when the constraints are attained. In turn, the dual minimizer can be written as a product of such a nondecreasing process and an optional strong supermartingale deflator. In the case of complete Brownian markets a similar result is proved in [HP93] and [EKJP98].

In order to implement the approach, we increase the dimensionality of the problem and treat as arguments of the indirect utility not only the initial wealth, but also the function that specifies the number of units of labor income (or the stream of liabilities) at any later time. This parametrization has the spirit of [HK04], however unlike [HK04, we go 
into (infinite-dimensional) non-reflexive spaces, which gives both novelty and technical difficulties to our analysis. Also, our formulation permits to price by marginal rate of substitution the whole labor income process. This is done through the subdifferentiability results in section 4. Note that the sub differential elements (prices) are time-dependent, so infinite-dimensional, unlike in [HK04].

Another contribution of the paper lies in the unified framework of admissibility outlined in section 2. More precisely, we assume that no-borrowing constraints are imposed starting from some pre-specified stopping time and hold up to the terminal time horizon. This framework allows us to treat in one formulation both the problem of no-borrowing constraints at all times (described above) and the one where borrowing against the future income is permitted with a constraint only at the end. The latter is well-studied in the literature, see [CSW01], [KŽ03], [HK04], [Ž05], and [Mos17]. In such a formulation, the constraints reduce to a single inequality and the decreasing processes in the dual feasible set become constants.

Among the many possibilities of constraints, Cuo97 considers the problem of investment and consumption with labor income and no-borrowing constraints in Brownian market, even allowing for incompleteness. The dual problem cannot be solved directly (in part because for these constraints the dual space considered is too small) but the primal can be solved with direct methods. An approximate dual sequence can then be recovered from the primal. We generalize [HP93] and [EKJP98] (complete Brownian markets) and Cuo97] (possibly incomplete Brownian markets) to the case of general semi-martingale incomplete markets. Our dual approach allows us, at the same time to obtain a dual characterization (complementary slackness) of the optimal consumption plan (not present in [Cuo97]), similar to the complete case in [HP93] and [EKJP98] and the possibility to study the dependence on labor income streams, through the parametrization of such streams.

Embedding path dependent problems into the convex duality framework have been analyzed in [Yu15], [XM16], [MP01, Section 3.3], whereas without duality but with random endowment it is considered in [Rás18], in the abstract singular control setting the duality approach is investigated in [BK17]. Our embedding does not require any condition on labor income replicability, which becomes highly technical in the presence of extra admissibility constraints. Even in the case where the only constraint is imposed at maturity, in this part our approach differs from the one in [HK04, where non-replicability of the endowment (in the appropriate sense) is used in the proofs as it ensures that the effective domain of the dual problem has the same dimensionality as the primal domain. Note that even if the labor income is spanned by the same sources of randomness as the stocks, the idea of replicating the labor income and then reducing the problem to the one without it, 
does not necessarily work under the borrowing constraints, see the discussion in [HP93, pp. 671-673].

Some of the more specific technical contributions of this paper can be summarized as follows:

- We analyze the boundary behavior of the value functions. Note that the value functions are defined over infinite-dimensional spaces.

- The finiteness of the indirect utilities without labor income is imposed only, as a necessary and sufficient condition that allows for the standard conclusions of the utility maximization theory, see [Mos15].

- We show existence-uniqueness results for the unbounded labor income both from above and below.

- We observe that the "Snell envelope proposition" [Kra96, Proposition 4.3] can be extended to the envelope over all stopping times that exceed a given initial stopping time $\theta_{0}$.

- We represent the dual value function in terms of uniformly integrable densities of martingale measures, i.e., the densities of martingale measures under which the maximal wealth process of a self-financing portfolio that superreplicates the labor income, is a uniformly integrable martingale, see Lemma 3.11 below.

Organization of the paper. In Section 2, we specify the model. We state and prove existence, uniqueness, semicontinuity and biconjugacy results in Section 3, subdifferentiability is proven in Section 4. Structure of the dual domain is analyzed in Section 5 and complimentary slackness is established in Section 6 .

\section{MODEL}

We consider a financial market model with finite time horizon $[0, T]$ and a zero interest rate. The price process $S=\left(S^{i}\right)_{i=1}^{d}$ of the stocks is assumed to be a semimartingale on a complete stochastic basis $\left(\Omega, \mathcal{F},\left(\mathcal{F}_{t}\right)_{t \in[0, T]}, \mathbb{P}\right)$, where $\mathcal{F}_{0}$ is trivial.

Let $(e)_{t \in[0, T]}$ be an optional process that specifies the labor income rate, which is assumed to follow a certain stochastic clock, that we specify below. Both processes $S$ and $e$ are given exogenously.

We define a stochastic clock as a nondecreasing, càdlàg, adapted process such that (finClock)

$$
\kappa_{0}=0, \mathbb{P}\left[\kappa_{T}>0\right]>0, \text { and } \kappa_{T} \leq A
$$

for some finite constant $A$. We note that the stochastic clock allows to include multiple standard formulations of the utility maximization problem in one formulation, see e.g., [Mos15, Example 2.5 - 2.9]. Let us define

$$
K_{t}:=\mathbb{E}\left[\kappa_{t}\right], \quad t \in[0, T]
$$


Remark 2.1. The function $K$ defined in (1) is right-continuous with left limits and takes values in $[0, A]$.

We assume the income and consumption are given in terms of the clock $\kappa$. Define a portfolio $\Pi$ as a quadruple $(x, q, H, c)$, where the constant $x$ is the initial value of the portfolio, the function $q:[0, T] \rightarrow \mathbb{R}$ is a bounded and Borel measurable function, which specifies the amount of labor income rate, $H=\left(H_{i}\right)_{i=1}^{d}$ is a predictable $S$-integrable process that corresponds to the amount of each stock in the portfolio, and $c=\left(c_{t}\right)_{t \in[0, T]}$ is the consumption rate, which we assume to be optional and nonnegative.

The wealth process $V=\left(V_{t}\right)_{t \in[0, T]}$ generated by the portfolio is

$$
V_{t}=x+\int_{0}^{t} H_{s} d S_{s}+\int_{0}^{t}\left(q_{s} e_{s}-c_{s}\right) d \kappa_{s}, \quad t \in[0, T]
$$

A portfolio $\Pi$ with $c \equiv 0$ and $q \equiv 0$ is called self-financing. The collection of nonnegative wealth processes generated by self-financing portfolios with initial value $x \geq 0$ is denoted by $\mathcal{X}(x)$, i.e.

$$
\mathcal{X}(x):=\left\{X \geq 0: X_{t}=x+\int_{0}^{t} H_{s} d S_{s}, t \in[0, T]\right\}, x \geq 0 .
$$

A probability measure $\mathbb{Q}$ is an equivalent local martingale measure if $\mathbb{Q}$ is equivalent to $\mathbb{P}$ and every $X \in \mathcal{X}(1)$ is a local martingale under $\mathbb{Q}$. We denote the family of equivalent local martingale measures by $\mathcal{M}$ and assume that

(noArb)

$\mathcal{M} \neq \emptyset$

This condition is equivalent to the absence of arbitrage opportunities in the market, see [DS94, DS98, as well as [KK07] for the exact statements and further references.

To rule out doubling strategies in the presence of random endowment, we need to impose additional restrictions. Following [DS97, we say that a nonnegative process in $\mathcal{X}(x)$ is maximal if its terminal value cannot be dominated by that of any other process in $\mathcal{X}(x)$. As in [DS97, we define an acceptable process to be a process of the form $X=X^{\prime}-X^{\prime \prime}$, where $X^{\prime}$ is a nonnegative wealth process generated by a self-financing portfolio and $X^{\prime \prime}$ is maximal.

Our unified framework of admissibility is given by a fixed stopping time $\theta_{0}$. The noborrowing constraints will hold starting at this stopping time until the end. Let $\Theta$ be the set of stopping times that are greater or equal than $\theta_{0}$.

Lemma 2.2. Let $q^{1}$ and $q^{2}$ be bounded, Borel measurable functions on $[0, T]$, such that $q^{1}=q^{2}$, dK-a.e. Then, the cumulative labor income processes $\int_{0}^{\cdot} q_{s}^{i} e_{s} d \kappa_{s}, i=1,2$, are indistinguishable. 
The proof of this lemma is given in section 3.1. Following [HK04, we denote by $\mathcal{X}(x, q)$ the set of acceptable processes with initial values $x$, that dominate the labor income on $\Theta$ :

$$
\begin{aligned}
\mathcal{X}(x, q):= & \left\{\text { acceptable } X: X_{0}=x\right. \text { and } \\
& \left.X_{\tau}+\int_{0}^{\tau} q_{s} e_{s} d \kappa_{s} \geq 0, \mathbb{P}-\text { a.s. for every } \tau \in \Theta\right\} .
\end{aligned}
$$

Let us set

$$
\mathcal{K}:=\{(x, q): \mathcal{X}(x, q) \neq \emptyset\}
$$

Let $\mathcal{K}$ denote the interior or $\mathcal{K}$ in the $\mathbb{R} \times \mathbb{L}^{\infty}(d K)$-norm topology. We characterize $\mathcal{K}$ in Lemma 3.2 below that in particular asserts that under Assumption $2.5, \mathcal{K} \neq \emptyset$.

The set of admissible consumptions is defined as

$$
\begin{aligned}
\mathcal{A}(x, q):= & \{\text { optional } c \geq 0: \text { there exists } X \in \mathcal{X}(x, q), \text { such that } \\
& \left.\int_{0}^{\tau} c_{s} d \kappa_{s} \leq X_{\tau}+\int_{0}^{\tau} q_{s} e_{s} d \kappa_{s}, \text { for every } \tau \in \Theta\right\}, \quad(x, q) \in \mathcal{K} .
\end{aligned}
$$

Note that $c \equiv 0$ belongs to $\mathcal{A}(x, q)$ for every $(x, q) \in \mathcal{K}$.

Remark 2.3. The no-borrowing constraints can also be written as

$$
\mathbb{P}\left(\int_{0}^{t} c_{s} d \kappa_{s} \leq X_{t}+\int_{0}^{t} q_{s} e_{s} d \kappa_{s}, \text { for every } \theta_{0} \leq t \leq T\right)=1
$$

We write the constraints in terms of stopping times $\tau \in \Theta$ as we use the stopping times $\tau \in \Theta$ (and the corresponding decreasing processes that jump from one to zero at these times) as the building blocks of our analysis.

Remark 2.4. It follows from Lemma 2.2, for every $x \in \mathbb{R}$, we have

$$
\mathcal{X}\left(x, q^{1}\right)=\mathcal{X}\left(x, q^{2}\right) \quad \text { and } \quad \mathcal{A}\left(x, q^{1}\right)=\mathcal{A}\left(x, q^{2}\right),
$$

where some of these sets might be empty

Hereafter, we shall impose the following conditions on the endowment process.

Assumption 2.5. There exists a maximal wealth process $X^{\prime}$ such that

$$
X_{t}^{\prime} \geq\left|e_{t}\right|, \quad \text { for every } t \in[0, T], \quad \mathbb{P} \text {-a.s.. }
$$

Moreover, Assumption 2.5 and (noArb imply that all the assertions of Lemma 3.2 hold.

Remark 2.6. If $\theta_{0}=\{T\}$, then Assumption 2.5 is equivalent to the assumptions on endowment in [HK04] (for the case of one-dimensional random endowment).

The preferences of an economic agent are modeled with a utility stochastic field $U=$ $U(t, \omega, x):[0, T] \times \Omega \times[0, \infty) \rightarrow \mathbb{R} \cup\{-\infty\}$. We assume that $U$ satisfies the conditions below. 
Assumption 2.7. For every $(t, \omega) \in[0, T] \times \Omega$, the function $x \rightarrow U(t, \omega, x)$ is strictly concave, increasing, continuously differentiable on $(0, \infty)$ and satisfies the Inada conditions:

$$
\lim _{x \downarrow 0} U^{\prime}(t, \omega, x)=\infty \quad \text { and } \quad \lim _{x \rightarrow \infty} U^{\prime}(t, \omega, x)=0,
$$

where $U^{\prime}$ denotes the partial derivative with respect to the third argument. At $x=0$ we suppose, by continuity, $U(t, \omega, 0)=\lim _{x \downarrow 0} U(t, \omega, x)$, which may be $-\infty$. For every $x \geq 0$ the stochastic process $U(\cdot, \cdot, x)$ is optional. Below, following the standard convention, we will not write $\omega$ in $U$.

The agent can control investment and consumption. The goal is to maximize expected utility. The value function $u$ is defined as:

$$
u(x, q):=\sup _{c \in \mathcal{A}(x, q)} \mathbb{E}\left[\int_{0}^{T} U\left(t, c_{t}\right) d \kappa_{t}\right], \quad(x, q) \in \mathcal{K} .
$$

In (2), we use the convention

$$
\mathbb{E}\left[\int_{0}^{T} U\left(t, c_{t}\right) d \kappa_{t}\right]:=-\infty \quad \text { if } \quad \mathbb{E}\left[\int_{0}^{T} U^{-}\left(t, c_{t}\right) d \kappa_{t}\right]=\infty .
$$

Here and below, $W^{-}$and $W^{+}$denote the negative and positive parts of a stochastic field $W$, respectively.

We employ duality techniques to obtain the standard conclusions of the utility maximization theory. We first define the convex conjugate stochastic field

$$
V(t, y):=\sup _{x>0}(U(t, x)-x y), \quad(t, y) \in[0, T] \times[0, \infty),
$$

and then observe that $-V$ satisfies Assumption 2.7. In order to construct the feasible set of the dual problem, we define the set $\mathcal{L}$ as the polar cone of $-\mathcal{K}$ :

$$
\mathcal{L}:=\left\{(y, r) \in \mathbb{R} \times \mathbb{L}^{1}(d K): x y+\int_{0}^{T} q_{s} r_{s} d K_{s} \geq 0 \text { for every }(x, q) \in \mathcal{K}\right\} .
$$

Remark 2.8. Under the conditions (finClock), (noArb) and Assumption (2.5), the set $\mathcal{L}$ is non-empty. By definition, it is closed in $\mathbb{R} \times \mathbb{L}^{1}(d K)$-norm and $\sigma\left(\mathbb{R} \times \mathbb{L}^{1}(d K), \mathbb{R} \times \mathbb{L}^{\infty}(d K)\right)$ topologies. Also, as shown later, the set $\mathcal{L}=(-\mathcal{K})^{o}$, i.e. the polar of $-\mathcal{K}$.

By $\mathcal{Z}$, we denote the set of càdlàg densities of equivalent local martingale measures:

$$
\mathcal{Z}:=\left\{\text { càdlàg }\left(\frac{d \mathbb{Q}_{t}}{d \mathbb{P}_{t}}\right)_{t \in[0, T]}: \quad \mathbb{Q} \in \mathcal{M}\right\} .
$$

Let us denote by $\mathbb{L}^{0}=\mathbb{L}^{0}(d \kappa \times \mathbb{P})$ the linear space of (equivalence classes of) real-valued optional processes on the stochastic basis $\left(\Omega, \mathcal{F},\left(\mathcal{F}_{t}\right)_{t \in[0, T]}, \mathbb{P}\right)$ which we equip with the 
topology of convergence in measure $(d \kappa \times \mathbb{P})$. For each $y \geq 0$ we define

$$
\mathcal{Y}(y):=\operatorname{cl}\{Y: Y \text { is càdlàg adapted and }
$$

$$
0 \leq Y \leq y Z(d \kappa \times \mathbb{P}) \text { a.e. for some } Z \in \mathcal{Z}\},
$$

where the closure is taken in $\mathbb{L}^{0}$. Now we are ready to set the domain of the dual problem:

$$
\begin{aligned}
\mathcal{Y}(y, r):=\{ & Y: Y \in \mathcal{Y}(y) \text { and } \\
& \mathbb{E}\left[\int_{0}^{T} c_{s} Y_{s} d \kappa_{s}\right] \leq x y+\int_{0}^{T} q_{s} r_{s} d K_{s}, \\
& \text { for every }(x, q) \in \mathcal{K} \text { and } c \in \mathcal{A}(x, q)\}
\end{aligned}
$$

Note that the definition (77) requires that every element of $\mathcal{Y}(y, r)$ is in $\mathcal{Y}(y), y \geq 0$. Also, for every $(y, r) \in \mathcal{L}, \mathcal{Y}(y, r) \neq \emptyset$, since $0 \in \mathcal{Y}(y, r)$.

We can now state the dual optimization problem:

$$
v(y, r):=\inf _{Y \in \mathcal{Y}(y, r)} \mathbb{E}\left[\int_{0}^{T} V\left(t, Y_{t}\right) d \kappa_{t}\right], \quad(y, r) \in \mathcal{L},
$$

where we use the convention:

$$
\mathbb{E}\left[\int_{0}^{T} V\left(t, Y_{t}\right) d \kappa_{t}\right]:=\infty \quad \text { if } \quad \mathbb{E}\left[\int_{0}^{T} V^{+}\left(t, Y_{t}\right) d \kappa_{t}\right]=\infty .
$$

Also, we set

$v(y, r):=\infty$ for $(y, r) \in \mathbb{R} \times \mathbb{L}^{1}(d K) \backslash \mathcal{L} \quad$ and $\quad u(x, q):=-\infty$ for $(x, q) \in \mathbb{R} \times \mathbb{L}^{\infty}(d K) \backslash \mathcal{K}$.

With this definition, it will be shown below in Theorem 3.1 that $u<\infty$ and $v>-\infty$ everywhere, so $u$ and $v$ are proper functions in the language of convex analysis. Let us recall that in the absence of random endowment, the dual value function is defined as

$$
\tilde{w}(y):=\inf _{Y \in \mathcal{Y}(y)} \mathbb{E}\left[\int_{0}^{T} V\left(t, Y_{s}\right) d \kappa_{s}\right], \quad y>0,
$$

whereas the primal value function is given by

$$
w(x):=u(x, 0), \quad x>0 .
$$

\section{Existence, uniqueness, And BiCONJUGACY}

Theorem 3.1. Let (finClock $)$ and (noArb), Assumptions 2.5 and 2.7 hold true and (finValue) $\quad w(x)>-\infty$ for every $x>0$ and $\widetilde{w}(y)<\infty$ for every $y>0$.

Then we have:

(i) $u$ is finite-valued on $\mathcal{K}$ and $u<\infty$ on $\mathbb{R} \times \mathbb{L}^{\infty}(d K)$. The dual value function $v$ satisfies $v>-\infty$ on $\mathbb{R} \times \mathbb{L}^{1}(d K)$, and the set $\{v<\infty\}$ is a nonempty convex subset of $\mathcal{L}$, whose closure in $\mathbb{R} \times \mathbb{L}^{1}(d K)$ equals to $\mathcal{L}$.

(ii) $u$ is concave, proper, and upper semicontinuous with respect to the norm-topology of $\mathbb{R} \times \mathbb{L}^{\infty}(d K)$ and the weak-star topology $\sigma\left(\mathbb{R} \times \mathbb{L}^{\infty}(d K), \mathbb{R} \times \mathbb{L}^{1}(d K)\right)$. For every 
$(x, q) \in\{u>-\infty\}$, there exists a unique solution to (2). Likewise, $v$ is convex, proper, and lower semicontinuous with respect to the norm-topology of $\mathbb{R} \times \mathbb{L}^{1}(d K)$ and the the weak topology $\sigma\left(\mathbb{R} \times \mathbb{L}^{1}(d K), \mathbb{R} \times \mathbb{L}^{\infty}(d K)\right)$. For every $(y, r) \in\{v<\infty\}$, there exists a unique solution to (8).

(iii) The functions $u$ and $v$ satisfy the biconjugacy relations

$$
\begin{aligned}
& u(x, q)=\inf _{(y, r) \in \mathcal{L}}\left(v(y, r)+x y+\int_{0}^{T} r_{s} q_{s} d K_{s}\right), \quad(x, q) \in \mathcal{K}, \\
& v(y, r)=\sup _{(x, q) \in \mathcal{K}}\left(u(x, q)-x y-\int_{0}^{T} r_{s} q_{s} d K_{s}\right), \quad(y, r) \in \mathcal{L} .
\end{aligned}
$$

Lemma 3.2. Let noArb and Assumption 2.5 hold. Then we have:

(i) for every $x>0,(x, 0)$ belongs to $\dot{\mathcal{K}}$ (in particular, $\stackrel{\mathcal{K}}{\mathbf{K}} \neq \emptyset$ ),

(ii) for every $q \in \mathbb{L}^{\infty}(d K)$, there exists $x>0$ such that $(x, q) \in \dot{\mathcal{K}}$,

(iii) $\sup _{\mathbb{Q} \in \mathcal{M}} \mathbb{E}^{\mathbb{Q}}\left[\int_{0}^{T}\left|e_{s}\right| d \kappa_{s}\right]<\infty$,

(iv) there exists a nonnegative maximal wealth process $X^{\prime \prime}$, such that

$$
X_{T}^{\prime \prime} \geq \int_{0}^{T}\left|e_{s}\right| d \kappa_{s}, \quad \mathbb{P}-\text { a.s. },
$$

$(v)$ there exists a nonnegative maximal wealth process $X^{\prime \prime}$, such that

$$
X_{t}^{\prime \prime} \geq \int_{0}^{t}\left|e_{s}\right| d \kappa_{s}, \quad t \in[0, T], \mathbb{P}-\text { a.s. }
$$

Proof. First, via [JS03, Proposition I.4.49] and (inClock), we get

$$
\begin{gathered}
\int_{0}^{T}\left|e_{s}\right| d \kappa_{s} \leq \int_{0}^{T} X_{s}^{\prime} d \kappa_{s}=-\int_{0}^{T} \kappa_{s-} d X_{s}^{\prime}+\kappa_{T} X_{T}^{\prime} \\
\leq-\int_{0}^{T} \kappa_{s-} d X_{s}^{\prime}+A X_{T}^{\prime}=A X_{0}^{\prime}+\int_{0}^{T}\left(A-\kappa_{s-}\right) d X_{s}^{\prime} .
\end{gathered}
$$

Therefore, the exists a self-financing wealth process $\bar{X}$, such that

$$
\int_{0}^{T}\left|e_{s}\right| d \kappa_{s} \leq \bar{X}_{T}
$$

Consequently, [DS97, Theorem 2.3] asserts the existence of a nonnegative maximal process $X^{\prime \prime}$, such that

$$
\int_{0}^{T}\left|e_{s}\right| d \kappa_{s} \leq X_{T}^{\prime \prime}
$$

i.e., (iv) holds. Therefore (iii) is valid as well, by [DS98, Theorem 5.12].

Relation (iv) and (noArb) imply $(v)$. To prove $(i)$ and $(i i)$, without loss of generality, we will suppose that in $(v), X_{0}^{\prime \prime}>0$. Let $q \in \mathbb{L}^{\infty}(d K)$ and $\varepsilon>0$ be fixed. Let us define

$$
x(q):=\left(\|q\|_{\mathbb{L}^{\infty}(d K)}+2 \varepsilon\right) X_{0}^{\prime \prime} .
$$

We claim that $(x(q), q) \in \stackrel{\circ}{\mathcal{K}}$. Let us consider arbitrary

$$
\left|x^{\prime}\right| \leq \varepsilon \quad \text { and } \quad q^{\prime} \in \mathbb{L}^{\infty}(d K):\left\|q^{\prime}\right\|_{\mathbb{L}^{\infty}(d K)} \leq \varepsilon,
$$


and set

$$
\tilde{X}_{t}:=\left(\|q\|_{\mathbb{L}^{\infty}(d K)}+2 \varepsilon+x^{\prime}\right) X_{t}^{\prime \prime}, \quad t \in[0, T] .
$$

Then, by item $(v)$, we have

$$
\tilde{X}_{t} \geq\left(\|q\|_{\mathbb{L}^{\infty}(d K)}+\varepsilon\right) X_{t}^{\prime \prime} \geq\left(\|q\|_{\mathbb{L}^{\infty}(d K)}+\varepsilon\right) \int_{0}^{t}\left|e_{s}\right| d \kappa_{s} \geq-\int_{0}^{t}\left(q_{s}+q_{s}^{\prime}\right)\left|e_{s}\right| d \kappa_{s} .
$$

We deduce that $\tilde{X} \in \mathcal{X}\left(\left(\|q\|_{\mathbb{L}^{\infty}(d K)}+2 \varepsilon+x^{\prime}\right) X_{0}^{\prime \prime}, q+q^{\prime}\right)$. In particular,

$$
\mathcal{X}\left(\left(\|q\|_{\mathbb{L}^{\infty}(d K)}+2 \varepsilon+x^{\prime}\right) X_{0}^{\prime \prime}, q+q^{\prime}\right) \neq \emptyset .
$$

As $x^{\prime}$ and $q^{\prime}$ are arbitrary elements satisfying (11), we deduce that $(x(q), q) \in \mathcal{K}$. This proves $(i i)$.

In order to show $(i)$, first we observe that $\mathcal{K}$ is a convex cone. Therefore, it suffices to prove that, for a given $\varepsilon>0$, we have

$$
\left(2 \varepsilon X_{0}^{\prime \prime}, 0\right) \in \stackrel{\circ}{\mathcal{K}}
$$

Again, let us consider $x^{\prime}$ and $q^{\prime}$ satisfying (11) and $\tilde{X}$ satisfying (12) for $q \equiv 0$. Then for every $t \in[0, T]$, we have

$$
\tilde{X}_{t} \geq \varepsilon X_{t}^{\prime \prime} \geq \varepsilon \int_{0}^{t}\left|e_{s}\right| d \kappa_{s} \geq-\int_{0}^{t} q_{s}^{\prime}\left|e_{s}\right| d \kappa_{s}
$$

Thus, $\mathcal{X}\left(\left(2 \varepsilon+x^{\prime}\right) X_{0}^{\prime \prime}, q^{\prime}\right) \neq \emptyset$. Consequently, as $x^{\prime}$ and $q^{\prime}$ are arbitrary elements satisfying (11), (13) holds and so is $(i)$. This completes the proof of the lemma.

Remark 3.3. A close look at the proofs shows that the conclusions of Theorem 3.1 also hold if instead of Assumption 2.5, we impose any of the equivalent assertions $($ iii $)-(v)$ of Lemma 3.2 .

3.1. Characterization of the primal and dual domains. The polar, $A^{o}$, of a nonempty subset $A$ of $\mathbb{R} \times \mathbb{L}^{\infty}(d K)$, is the subset of $\mathbb{R} \times \mathbb{L}^{1}(d K)$, defined by

$$
A^{o}:=\left\{(y, r) \in \mathbb{R} \times \mathbb{L}^{1}(d K): x y+\int_{0}^{T} q_{s} r_{s} d K_{s} \leq 1, \quad \text { for every }(x, q) \in A\right\} .
$$

The polar of a subset of $\mathbb{R} \times \mathbb{L}^{1}(d K)$ is defined similarly.

Proposition 3.4. Under Assumption 2.5 and conditions (finClock and (noArb), we have:

(i) Let $(x, q) \in \mathbb{R} \times \mathbb{L}^{\infty}(d K)$. Then $c \in \mathcal{A}(x, q)$ (thus, $\mathcal{A}(x, q) \neq \emptyset$ so $\left.(x, q) \in \mathcal{K}\right)$ if and only if

$$
\mathbb{E}\left[\int_{0}^{T} c_{s} Y_{s} d \kappa_{s}\right] \leq x y+\int_{0}^{T} q_{s} r_{s} d K_{s}, \text { for every }(y, r) \in \mathcal{L} \text { and } Y \in \mathcal{Y}(y, r) .
$$


(ii) Likewise, for $(y, r) \in \mathbb{R} \times \mathbb{L}^{1}(d K)$ we have $(y, r) \in \mathcal{L}$ and $Y \in \mathcal{Y}(y, r)$ if and only if $\mathbb{E}\left[\int_{0}^{T} c_{s} Y_{s} d \kappa_{s}\right] \leq x y+\int_{0}^{T} q_{s} r_{s} d K_{s}$, for every $(x, q) \in \mathcal{K}$ and $c \in \mathcal{A}(x, q)$.

We also have $\mathcal{K}=(-\mathcal{L})^{o}=\mathrm{cl} \dot{\mathcal{K}}$, where the closure is taken both in norm $\mathbb{R} \times \mathbb{L}^{1}(d K)$ and $\sigma\left(\mathbb{R} \times \mathbb{L}^{\infty}(d K), \mathbb{R} \times \mathbb{L}^{1}(d K)\right)$ topologies.

Remark 3.5. It follows from Proposition 3.4 that for every $(x, q) \in \mathcal{K}, \mathcal{A}(x, q) \neq \emptyset$ as $0 \in \mathcal{A}(x, q)$. Likewise, for every $(y, r) \in \mathcal{L}, \mathcal{Y}(y, r) \neq \emptyset$ as $0 \in \mathcal{Y}(y, r)$. Moreover, for every $(x, q) \in \dot{\mathcal{K}}$, each of the sets $\mathcal{A}(x, q)$ and $\quad \bigcup \quad \mathcal{Y}(y, r)$ contain a strictly positive element, see Lemma 3.14 below.

The proof of Proposition 3.4 will be given via several lemmas. Let

$\mathcal{M}^{\prime}$ be the set of equivalent local martingale measures, under which

$X^{\prime \prime}$ (from Lemma 3.2, item $(v)$ ) is a uniformly integrable martingale.

Note that by [DS97, Theorem 5.2], $\mathcal{M}^{\prime}$ is a nonempty, convex subset of $\mathcal{M}$, which is also dense in $\mathcal{M}$ in the total variation norm.

Remark 3.6. Even though the results in DS97 are obtained under the condition that $S$ is a locally bounded process, they also hold without local boundedness assumption, see the discussion in [HKS05, Remark 3.4].

Let $Z^{\prime}$ denote the set of the corresponding càdlàg densities, i.e.,

$$
\mathcal{Z}^{\prime}:=\left\{\text { càdlàg } Z: Z_{t}=\mathbb{E}\left[\frac{d \mathbb{Q}}{d \mathbb{P}} \mid \mathcal{F}_{t}\right], t \in[0, T], \mathbb{Q} \in \mathcal{M}^{\prime}\right\} .
$$

We also set

$$
\Upsilon:=\left\{1_{[0, \tau]}(t), t \in[0, T]: \tau \in \Theta\right\} .
$$

Lemma 3.7. Let the conditions of Proposition 3.4 hold, $\mathbb{Q} \in \mathcal{M}^{\prime}, Z=Z^{\mathbb{Q}}$ be the corresponding element of $\mathcal{Z}^{\prime}$, and $\Lambda \in \Upsilon$. Then there exists $r \in \mathbb{L}^{1}(d K)$, uniquely defined by

$$
\int_{0}^{t} r_{s} d K_{s}=\mathbb{E}\left[\int_{0}^{t} \Lambda_{s} Z_{s} e_{s} d \kappa_{s}\right], \quad t \in[0, T],
$$

such that $(1, r) \in \mathcal{L}$ and $Z \Lambda=\left(Z_{t} \Lambda_{t}\right)_{t \in[0, T]} \in \mathcal{Y}(1, r)$.

Proof. Let $(x, q) \in \mathcal{K}$ and $c \in \mathcal{A}(x, q)$. Then there exists $X \in \mathcal{X}(x, q)$, such that

$$
\int_{0}^{\tau} c_{s} d \kappa_{s} \leq X_{\tau}+\int_{0}^{\tau} q_{s} e_{s} d \kappa_{s}, \quad \text { for every } \tau \in \Theta
$$

In particular, (17) holds for the particular $\tau$, such that $\Lambda_{t}=1_{[0, \tau]}(t), t \in[0, T]$. Let $G_{t}:=\int_{0}^{t}\left(q_{s} e_{s}\right)^{+} d \kappa_{s}, t \in[0, T]$, where $(\cdot)^{+}$denotes the positive part. By [Pro04, Theorem III.29, p. 128], $\int_{0}^{t} G_{s-} d Z_{s}, t \in[0, T]$, is a local martingale, so let $\left(\sigma_{n}\right)_{n \in \mathbb{N}}$ be its localizing 
sequence. Then by the monotone convergence theorem, integration by parts formula, and the optional sampling theorem, we get

$$
\begin{aligned}
& \mathbb{E}\left[\int_{0}^{T} Z_{s}\left(q_{s} e_{s}\right)^{+} \Lambda_{s} d \kappa_{s}\right]=\lim _{n \rightarrow \infty} \mathbb{E}\left[\int_{0}^{\sigma_{n} \wedge \tau} Z_{s} d G\right] \\
= & \lim _{n \rightarrow \infty} \mathbb{E}\left[Z_{\sigma_{n} \wedge \tau} G_{\sigma_{n} \wedge \tau}\right]-\lim _{n \rightarrow \infty} \mathbb{E}\left[\int_{0}^{\sigma_{n} \wedge \tau} G_{s-} d Z_{s}\right] \\
= & \lim _{n \rightarrow \infty} \mathbb{E}\left[Z_{\sigma_{n} \wedge \tau} G_{\sigma_{n} \wedge \tau}\right]=\lim _{n \rightarrow \infty} \mathbb{E}^{\mathbb{Q}}\left[G_{\sigma_{n} \wedge \tau}\right]=\mathbb{E}^{\mathbb{Q}}\left[G_{\tau}\right],
\end{aligned}
$$

where in the last equality we used the monotone convergence theorem again. Here finiteness of $\mathbb{E}^{\mathbb{Q}}\left[G_{\tau}\right]=\mathbb{E}^{\mathbb{Q}}\left[\int_{0}^{\tau}\left(q_{s} e_{s}\right)^{+} d \kappa_{s}\right]$ follows from Assumption 2.5 via Lemma 3.2, part (iii). In a similar manner, we can show that

$$
\begin{aligned}
\mathbb{E}\left[\int_{0}^{T}\left(q_{s} e_{s}\right)^{-} Z_{s} \Lambda_{s} d \kappa_{s}\right] & =\mathbb{E}^{\mathbb{Q}}\left[\int_{0}^{\tau}\left(q_{s} e_{s}\right)^{-} d \kappa_{s}\right]<\infty \\
\mathbb{E}\left[\int_{0}^{T} c_{s} Z_{s} \Lambda_{s} d \kappa_{s}\right] & =\mathbb{E}^{\mathbb{Q}}\left[\int_{0}^{\tau} c_{s} d \kappa_{s}\right]<\infty .
\end{aligned}
$$

[HK04, Lemma 4] applies here and asserts that $X$ in (17) is a supermartingale under $\mathbb{Q}$. Therefore, from (17), using (18) and (19), and taking expectation under $\mathbb{Q}$, we get

$$
\mathbb{E}\left[\int_{0}^{T} c_{s} \Lambda_{s} Z_{s} d \kappa_{s}\right] \leq x+\mathbb{E}\left[\int_{0}^{T} q_{s} e_{s} Z_{s} \Lambda_{s} d \kappa_{s}\right]
$$

Let us define

$$
R_{t}:=\mathbb{E}\left[\int_{0}^{t} \Lambda_{s} Z_{s} e_{s} d \kappa_{s}\right], \quad t \in[0, T] .
$$

Using the monotone class theorem, we obtain

$$
\mathbb{E}\left[\int_{0}^{T} \tilde{q}_{s} e_{s} Z_{s} \Lambda_{s} d \kappa_{s}\right]=\int_{0}^{T} \tilde{q}_{s} d R_{s}, \quad \text { for every } \quad \tilde{q} \in \mathbb{L}^{\infty}(d K) .
$$

We claim that $d R$ is absolutely continuous with respect to $d K$. First, using the $\pi-\lambda$ theorem, one can show that for every Borel-measurable subset $A$ of $[0, T]$, we have

$$
K(A)=\mathbb{E}\left[\int_{0}^{T} 1_{A}(t) d \kappa_{t}\right] \quad \text { and } \quad R(A)=\mathbb{E}\left[\int_{0}^{T} 1_{A}(t) \Lambda_{t} Z_{t} e_{t} d \kappa_{t}\right] .
$$

Thus, if for some $A, K(A)=0$, then $\int_{0}^{T} 1_{A}(t) d \kappa_{t}=0$ a.s. and $\kappa_{t}^{A}:=\int_{0}^{t} 1_{A}(s) d \kappa_{s}, t \in[0, T]$, satisfies $\kappa_{T}^{A}=0$ a.s. and $\int_{0}^{T} \Lambda_{t} Z_{t} e_{t} d \kappa_{t}^{A}=\int_{0}^{T} \Lambda_{t} Z_{t} e_{t} 1_{A}(t) d \kappa_{t}=0$ a.s.

As $d R$ is absolutely continuous with respect to $d K$, there exists a unique $r \in \mathbb{L}^{1}(d K)$, such that (16) holds. Since the left-hand side in (20) is nonnegative and since $(x, q)$ is an arbitrary element of $\mathcal{K}$, we deduce from the definition of $\mathcal{L}$, (4), that $(1, r) \in \mathcal{L}$. Finally, it follows from (20) and (21) that $Z \Lambda \in \mathcal{Y}(1, r)$. This completes the proof of the lemma.

Remark 3.8. The natural convexification of the set $\Upsilon$ consists of non-negative left-continuous decreasing and adapted processes $D$ such that $D_{\theta_{0}}=1$. In the context of utilitymaximization constraints (and for $\theta_{0}=0$ ), this is follows from [HP93] and [EKJP98. We investigate convexification of the constraints in the later Sections 5 and 6 , where will 
extend Lemma 3.7 to a more general set of decreasing processes than $\Lambda$ that drives the dual domain and that allows for the multiplicative decomposition of the dual minimizer.

Corollary 3.9. Let the conditions of Proposition 3.4 hold, $\mathbb{Q} \in \mathcal{M}^{\prime}, Z$ be the cádlág modification of the density process $\mathbb{E}\left[\frac{d \mathbb{Q}}{d \mathbb{P}} \mid \mathcal{F}_{t}\right], t \in[0, T]$. Then there exists $r \in \mathbb{L}^{1}(d K)$, such that $Z \in \mathcal{Y}(1, r)$, where

$$
\int_{0}^{t} r_{s} d K_{s}=\mathbb{E}\left[\int_{0}^{t} Z_{s} e_{s} d \kappa_{s}\right], \quad t \in[0, T],
$$

and $(1, r) \in \mathcal{L}$.

Proof of Lemma 2.2. Let us fix $\mathbb{Q} \in \mathcal{M}^{\prime}$ and let $Z \in \mathcal{Z}^{\prime}$ be the corresponding density process. As in Lemma 3.7, we can show that there exists $r \in \mathbb{L}^{1}(d K)$, such that for every bounded and Borel measurable function $q$ on $[0, T]$ we have

$$
\int_{0}^{t} q_{s} r_{s} d K_{s}=\mathbb{E}\left[\int_{0}^{t} q_{s} Z_{s}\left|e_{s}\right| d \kappa_{s}\right], \quad t \in[0, T] .
$$

Let $\bar{q}:=\left|q^{1}-q^{2}\right|$, then as $\bar{q}=0, d K$-a.e., we get

$$
\mathbb{E}\left[\int_{0}^{T} \bar{q}_{s}\left|e_{s}\right| Z_{s} d \kappa_{s}\right]=\int_{0}^{T} \bar{q}_{s} r_{s} d K=0 .
$$

Therefore, using integration by parts and via (22), we obtain

$$
0=\int_{0}^{T} \bar{q}_{s} r_{s} d K=\mathbb{E}\left[\int_{0}^{T} q_{s} Z_{s}\left|e_{s}\right| d \kappa_{s}\right]=\mathbb{E}^{\mathbb{Q}}\left[\int_{0}^{T} q_{s}\left|e_{s}\right| d \kappa_{s}\right] .
$$

Consequently, $\int_{0}^{T} q_{s}\left|e_{s}\right| d \kappa_{s}=0, \mathbb{Q}$-a.s., and by the equivalence of $\mathbb{Q}$ and $\mathbb{P}$, also $\mathbb{P}$-a.s. As, by construction $\int_{0}^{t} q_{s}\left|e_{s}\right| d \kappa_{s}=0, t \in[0, T]$, is a nonnegative and non-decreasing process, whose terminal value is $0, \mathbb{P}$-a.s., we conclude that it is indistinguishable from the 0 -valued process. The assertions of the lemma follows.

Lemma 3.10. Let the conditions of Proposition 3.4 hold, $(x, q) \in \mathcal{K}$, and $c$ is a nonnegative optional process. Then $c \in \mathcal{A}(x, q)$ if and only if

$$
\mathbb{E}\left[\int_{0}^{T} c_{s} \Lambda_{s} Z_{s} d \kappa_{s}\right] \leq x+\mathbb{E}\left[\int_{0}^{T} q_{s} e_{s} \Lambda_{s} Z_{s} d \kappa_{s}\right]=x+\int_{0}^{T} q_{s} r_{s} d K_{s},
$$

for every $Z \in \mathcal{Z}^{\prime}$ and $\Lambda \in \Upsilon$, where $r$ is given by (16).

Proof. Let $c \in \mathcal{A}(x, q)$. Then for every $Z \in \mathcal{Z}^{\prime}$ and $\Lambda \in \Upsilon$, the validity of (23) follows from the definition of $\mathcal{A}(x, q)$, integration by parts formula and supermartingale property of every $X \in \mathcal{X}(x, q)$ under every $\mathbb{Q} \in \mathcal{M}^{\prime}$, which in turn follows from [HK04, Lemma 4].

Conversely, let (23) holds for every $Z \in \mathcal{Z}^{\prime}$ and $\Lambda \in \Upsilon$. Then, we have

$$
\mathbb{E}\left[\int_{0}^{T}\left(c_{s}-q_{s} e_{s}\right) Z_{s} \Lambda_{s} d \kappa_{s}\right] \leq x
$$


which, in view of the definition of $\Upsilon$ in (15), localization, and integration by parts, implies that

$$
\sup _{\mathbb{Q} \in \mathcal{M}^{\prime}, \tau \in \Theta} \mathbb{E}^{\mathbb{Q}}\left[\int_{0}^{\tau}\left(c_{s}-q_{s} e_{s}\right) d \kappa_{s}\right] \leq x,
$$

For $X^{\prime \prime}$ given by Lemma 3.2, item $(v)$, let us denote

$$
f_{t}:=\|q\|_{\mathbb{L}^{\infty}(d K)} X_{t}^{\prime \prime}+\int_{0}^{t}\left(c_{s}-q_{s} e_{s}\right) d \kappa_{s}, \quad t \in[0, T] .
$$

It follows from Assumption 2.5 and item $(v)$ of Lemma 3.2 that $f$ is a nonnegative process. We observe that the proof of [Kra96, Proposition 4.3] goes through, if we only take stopping times in $\Theta$ and measures in $\mathcal{M}^{\prime}$. This proposition allows to conclude that there exists a nonnegative càdlàg process $V$, such that

$$
V_{t}=\operatorname{esssup}_{\tau \in \Theta: \tau \geq t, \mathbb{Q} \in \mathcal{M}^{\prime}} \mathbb{E}^{\mathbb{Q}}\left[f_{\tau} \mid \mathcal{F}_{t}\right], \quad t \in[0, T],
$$

which is a supermartingale for every $\mathbb{Q} \in \mathcal{M}^{\prime}$. Therefore, by the density of $\mathcal{M}^{\prime}$ in $\mathcal{M}$ in the norm topology of $\mathbb{L}^{1}(\mathbb{P})$ and Fatou's lemma, $V$ is a supermartingale under every $\mathbb{Q} \in \mathcal{M}$. Moreover, $V_{0}$ satisfies

$$
V_{0} \leq x+\|q\|_{\mathbb{L}^{\infty}(d K)} X_{0}^{\prime \prime}
$$

by (26), (24), and by following the argument in the proof of Lemma 3.7 .

We would like to apply the optional decomposition theorem of Fölmer and Kramkov, [FK97, Theorem 3.1]. For this, we need to show that $V$ is a local supermartingale under every $\mathbb{Q}$, such that every $X \in \mathcal{X}(1)$ is a $\mathbb{Q}$-local supermartingale. However, $\mathcal{M}$ is dense in the set of such measures in the norm topology of $\mathbb{L}^{1}(\mathbb{P})$, by the results of Delbaen and Schachermayer, see [DS98, Proposition 4.7]. Therefore, the supermartingale property of $V$ under every such $\mathbb{Q}$ follows from Fatou's lemma and supermartingale property of $V$ under every $\mathbb{Q} \in \mathcal{M}$ established above. Therefore, by [FK97, Theorem 3.1], we get

$$
V_{t}=V_{0}+H \cdot S_{t}-A_{t}, \quad t \in[0, T]
$$

where $A$ is a nonnegative increasing process that starts at 0 . Subtracting the constant $\|q\|_{\mathbb{L}^{\infty}(d K)} X_{0}^{\prime \prime}$ from both sides of (25), we get

$$
\begin{aligned}
& \int_{0}^{\tau}\left(c_{s}-q_{s} e_{s}\right) d \kappa_{s}=f_{\tau}-\|q\|_{\mathbb{L}^{\infty}(d K)} X_{0}^{\prime \prime} \leq V_{\tau}-\|q\|_{\mathbb{L}^{\infty}(d K)} X_{0}^{\prime \prime} \\
& =V_{0}-\|q\|_{\mathbb{L}^{\infty}(d K)} X_{0}^{\prime \prime}+H \cdot S_{\tau}-A_{\tau} \leq x+H \cdot S_{\tau}, \quad \tau \in \Theta,
\end{aligned}
$$

where $x+H \cdot S$ is acceptable, since $V$ is nonnegative. Consequently, $c \in \mathcal{A}(x, q)$. This completes the proof of the lemma.

Proof of Proposition 3.4. The assertions of item $(i)$ follow from Lemma 3.10. It remains to show that the affirmations of item $(i i)$ hold. Fix a $(y, r) \in \mathcal{L}$. If $Y \in \mathcal{Y}(y, r),(i i)$ 
follows from the definition of $\mathcal{Y}(y, r)$. Conversely, if $(i i)$ holds for a nonnegative process $Y$, then since $(x, 0) \in \mathcal{K}$ for every $x>0$, we have

$$
\mathbb{E}\left[\int_{0}^{T} c_{s} Y_{s} d \kappa_{s}\right] \leq 1 \quad \text { for every } c \in \mathcal{A}\left(\frac{1}{y}, 0\right) .
$$

Via [Mos15, Proposition 4.4], we deduce that $Y \in \mathcal{Y}(y)$ and is such that (ii) holds. Therefore, $Y \in \mathcal{Y}(y, r)$.

We have $\mathcal{K} \neq \emptyset$, where the interior is taken with respect to the norm-topology. According to Proposition 3.4 (i) we have also $\mathcal{K}=(-\mathcal{L})^{o}$. The set $\mathcal{K}$, as the polar of $\mathcal{L}$, is convex and closed both in (strong) $\mathbb{R} \times \mathbb{L}^{\infty}(d K)$ and $\sigma\left(\mathbb{R} \times \mathbb{L}^{\infty}(d K), \mathbb{R} \times \mathbb{L}^{1}(d K)\right)$ topologies. Having non-empty strong interior, we obtain $\mathcal{K}=\mathrm{cl} \mathcal{K}$ where the closure is in the strong-topology. Since $\mathcal{K}$ is also closed in the weaker $\sigma\left(\mathbb{R} \times \mathbb{L}^{\infty}(d K), \mathbb{R} \times \mathbb{L}^{1}(d K)\right)$ topology we obtain that

$$
\mathcal{K}=(-\mathcal{L})^{o}=\mathrm{cl} \stackrel{\circ}{\mathcal{K}}
$$

where the closure is taken in both topologies.

3.2. Preliminary properties of the value functions, in particular finiteness. Let $\mathbf{L}_{+}^{0}$ be the positive orthant of $\mathbf{L}^{0}$. The polar of a set $A \subseteq \mathbf{L}_{+}^{0}$ is defined as

$$
A^{o}:=\left\{c \in \mathbf{L}_{+}^{0}: \mathbb{E}\left[\int_{0}^{T} c_{s} Y_{s} d \kappa_{s}\right] \leq 1, \quad \text { for every } Y \in A\right\} .
$$

We recall that the sets $\mathcal{Z}$ and $\mathcal{Z}^{\prime}$ are defined in (5) and (14), respectively.

Lemma 3.11. Under the conditions of Theorem 3.1, we have

$$
\left(\mathcal{Z}^{\prime}\right)^{o o}=\mathcal{Y}(1)
$$

and

$$
\tilde{w}(y)=\inf _{Y \in \mathcal{Z}^{\prime}} \mathbb{E}\left[\int_{0}^{T} V\left(t, y Y_{s}\right) d \kappa_{s}\right]<\infty, \quad y>0 .
$$

Proof. It follows from [DS97, Theorem 5.2] that $\mathcal{Z}^{\prime}$ is dense in $\mathcal{Z}$ in $\mathbf{L}^{0}$. It follows from Fatou's lemma that

$$
\left(\mathcal{Z}^{\prime}\right)^{o}=\mathcal{Z}^{o}
$$

Therefore, [Mos15, Lemma 4.2 and Proposition 4.4] imply (27).

One can show that $\mathcal{Z}^{\prime}$ is closed under countable convex combinations, where the martingale property follows from the monotone convergence theorem and the càdlàg structure of the limit is guaranteed by [DM82, Theorem VI.18], see also [KLPO14, Proposition 5.1] for more details in similar settings. Now, (28) follows (up to a notational change) from [Mos15, Theorem 3.3]. This completes the proof of the lemma. 
Lemma 3.12. Under the conditions of Theorem 3.1, for every $(x, q) \in \mathcal{K}$ and $(y, r) \in \mathcal{L}$, we have

$$
u(x, q) \leq v(y, r)+x y+\int_{0}^{T} r_{s} q_{s} d K_{s} .
$$

Proof. Fix an arbitrary $(x, q) \in \operatorname{cl} \mathcal{K}, c \in \mathcal{A}(x, q)$ as well as $(y, r) \in \mathcal{L}, Y \in \mathcal{Y}(y, r)$. Using Proposition 3.4 and (3), we get

$$
\begin{aligned}
\mathbb{E}\left[\int_{0}^{T} U\left(t, c_{s}\right) d \kappa_{s}\right] & \leq \mathbb{E}\left[\int_{0}^{T} U\left(t, c_{s}\right) d \kappa_{s}\right]+x y+\int_{0}^{T} r_{s} q_{s} d K_{s}-\mathbb{E}\left[\int_{0}^{T} c_{s} Y_{s} d \kappa_{s}\right] \\
& \leq \mathbb{E}\left[\int_{0}^{T} V\left(t, Y_{s}\right) d \kappa_{s}\right]+x y+\int_{0}^{T} r_{s} q_{s} d K_{s} .
\end{aligned}
$$

This implies the assertion of the lemma.

For every $(x, q)$ in $\mathcal{K}$, we define

$$
\begin{aligned}
\mathcal{B}(x, q) & :=\left\{(y, r) \in \mathcal{L}: x y+\int_{0}^{T} r_{s} q_{s} d K_{s} \leq 1\right\} \\
\mathcal{D}(x, q) & :=\bigcup_{(y, r) \in \mathcal{B}(x, q)} \mathcal{Y}(y, r) .
\end{aligned}
$$

The subsequent lemma established boundedness of $\mathcal{B}(x, q)$ for $(x, q)$ in $\mathcal{K}$ in $\mathbb{R} \times \mathbb{L}^{1}(d K)$.

Lemma 3.13. Under the conditions of Theorem [3.1, for every $(x, q) \in \dot{\mathcal{K}}, \mathcal{B}(x, q)$ is bounded in $\mathbb{R} \times \mathbb{L}^{1}(d K)$.

Proof. Fix an $(x, q) \in \dot{\mathcal{K}}$. Then there exists $\varepsilon>0$, such that for every

$$
\left|x^{\prime}\right| \leq \varepsilon \quad \text { and } \quad\left\|q^{\prime}\right\|_{\mathbb{L}^{\infty}} \leq \varepsilon,
$$

we have $\left(x+x^{\prime}, q+q^{\prime}\right) \in \dot{\mathcal{K}}$. Let us fix an arbitrary $(y, r) \in \mathcal{B}(x, q)$. Then for every $\left(x^{\prime}, q^{\prime}\right)$ satisfying (30), by the definitions of $\mathcal{L}$ and $\mathcal{B}(x, q)$, respectively, we get

$$
\begin{aligned}
x y+\int_{0}^{T} q_{s} r_{s} d K_{s}+x^{\prime} y+\int_{0}^{T} q_{s}^{\prime} r_{s} d K_{s} & \geq 0, \\
x y+\int_{0}^{T} q_{s} r_{s} d K_{s} & \leq 1,
\end{aligned}
$$

which implies that

$$
-x^{\prime} y-\int_{0}^{T} q_{s}^{\prime} r_{s} d K_{s} \leq x y+\int_{0}^{T} q_{s} r_{s} d K_{s} \leq 1
$$

Taking

$$
x^{\prime}=-\varepsilon \quad \text { and } \quad q^{\prime} \equiv 0,
$$

we deduce from (31) that $y \leq \frac{1}{\varepsilon}$. Also, by the definition of $\mathcal{L}$ and Lemma 3.2, item $(i)$, $y \geq 0$. In turn, setting

$$
x^{\prime}=0 \quad \text { and } \quad q^{\prime}=-\varepsilon 1_{\{r \geq 0\}}+\varepsilon 1_{\{r<0\}},
$$

we obtain from (31) that $\|r\|_{\mathbb{L}^{1}} \leq \frac{1}{\varepsilon}$. This completes the proof of the lemma. 
Lemma 3.14. Let the conditions of Theorem 3.1 hold and $(x, q)$ be an arbitrary element of $\mathcal{K}$. Then, we have:

(i) $\mathcal{A}(x, q)$ contains a strictly positive process.

(ii) The constant $\bar{y}(x, q)$ given by

$$
\bar{y}(x, q):=\frac{1}{|x|+\|q\|_{\mathbb{L}^{\infty}(d K)} \sup _{\mathbb{Q} \in \mathcal{M}} \mathbb{E}^{\mathbb{Q}}\left[\int_{0}^{T}\left|e_{s}\right| d \kappa_{s}\right]},
$$

takes values in $(0, \infty)$ and satisfies

$$
\bar{y}(x, q) \mathcal{Z}^{\prime} \subseteq \mathcal{D}(x, q) .
$$

In particular, for every $z>0, z \mathcal{D}(x, q)$ contains a strictly positive process $Y$ such that

$$
\mathbb{E}\left[\int_{0}^{T} V\left(s, Y_{s}\right) d \kappa_{s}\right]<\infty .
$$

Proof. In order to show $(i)$, we observe that the existence of a positive process in $\mathcal{A}(x, q)$ follows from the fact that $(x-\delta, q) \in \mathcal{K}$ for a sufficiently small $\delta$. Now the constant-valued consumption $\delta / A>0$, where $A$ is the constant that dominates the terminal value of the stochastic clock $\kappa$ in (finClock $)$, is in $\mathcal{A}(x, q)$.

In order to prove $(i i)$, let us consider $\bar{y}(x, q)$ given by (32). It follows from Lemma 3.2. item $($ iii $)$, that $\bar{y}(x, q) \in(0, \infty)$. For this $\bar{y}(x, q)$, using Corollary 3.9, one can show (33). This and Lemma 3.11 (note that finiteness of $\tilde{w}$ follows directly from (finValue) ) imply that for every $z>0$, there exists a positive $Y \in z \mathcal{D}(x, q)$, such that (34) holds.

Lemma 3.15. Under the conditions of Theorem [3.1, for every $(x, q) \in \dot{\mathcal{K}}$ we have

$$
-\infty<u(x, q)<\infty
$$

and $u<\infty$ on $\mathbb{R} \times \mathbb{L}^{\infty}(d K)$.

Proof. Let us fix an arbitrary $(x, q) \in \mathcal{K}$. Since $\mathcal{K}$ is an open convex cone, there exists $\lambda \in(0,1),\left(x_{1}, q_{1}\right) \in \dot{\mathcal{K}}$, and $x_{2}>0$, such that

$$
(x, q)=\lambda\left(x_{1}, q_{1}\right)+(1-\lambda)\left(x_{2}, 0\right) .
$$

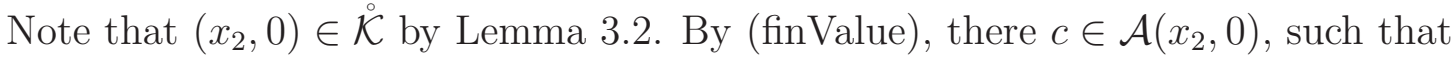

$$
\mathbb{E}\left[\int_{0}^{T} U\left(t,(1-\lambda) c_{t}\right) d \kappa_{t}\right]>-\infty
$$

As $\mathcal{A}\left(x_{1}, q_{1}\right) \neq \emptyset$ (see Remark 3.5), there exists $\tilde{c} \in \mathcal{A}\left(x_{1}, q_{1}\right)$. As $U(t, \cdot)$ is nondecreasing, we get

$$
u(x, q) \geq \mathbb{E}\left[\int_{0}^{T} U\left(t, \lambda \tilde{c}_{t}+(1-\lambda) c_{t}\right) d \kappa_{t}\right] \geq \mathbb{E}\left[\int_{0}^{T} U\left(t,(1-\lambda) c_{t}\right) d \kappa_{t}\right]>-\infty
$$


where the last inequality follows from (35). This implies finiteness of $u$ on $\mathcal{K}$ from below.

In order to show finiteness from above, let us fix a process $c \in \mathcal{A}(x, q)$, such that

$$
\mathbb{E}\left[\int_{0}^{T} U\left(t, c_{t}\right) d \kappa_{t}\right]>-\infty
$$

By Lemma 3.11, there exists $Y \in \mathcal{Z}^{\prime}$, such that

$$
\mathbb{E}\left[\int_{0}^{T} V\left(t, Y_{t}\right) d \kappa_{t}\right]<\infty .
$$

It follows from Lemma 3.7 that $Y \in \mathcal{Y}(1, \rho)$ for some $(1, \rho) \in \mathcal{L}$. Therefore, by Proposition 3.4, we get

$$
\begin{aligned}
\mathbb{E}\left[\int_{0}^{T} U\left(s, c_{s}\right) d \kappa_{s}\right] & \leq \mathbb{E}\left[\int_{0}^{T} U\left(s, c_{s}\right) d \kappa_{s}\right]+x+\int_{0}^{T} \rho_{s} q_{s} d K_{s}-\mathbb{E}\left[\int_{0}^{T} c_{s} Y_{s} d \kappa_{s}\right] \\
& \leq \mathbb{E}\left[\int_{0}^{T} V\left(s, Y_{s}\right) d \kappa_{s}\right]+x+\int_{0}^{T} \rho_{s} q_{s} d K_{s} .
\end{aligned}
$$

As $Y$ satisfies (36), we conclude that $u(x, q)<\infty$. Moreover, for $(x, q) \in \mathcal{K}$, as $\mathcal{A}(x, q) \neq \emptyset$ by Remark 3.5, every $c \in \mathcal{A}(x, q)$ satisfies (37) (with the same $Y$ ). This implies that $u<\infty$ on $\mathcal{K}$ and therefore, by (9), on $\mathbb{R} \times \mathbb{L}^{\infty}(d K)$. This completes the proof of the lemma.

We recall that, for every $(x, q) \in \mathcal{K}, \mathcal{D}(x, q)$ is defined in (29). Let $c l \mathcal{D}(x, q)$ denote the closure of $\mathcal{D}(x, q)$ in $\mathbb{L}^{0}(d \kappa \times \mathbb{P})$. The following lemma proves a delicate point that, for $(x, q) \in \mathcal{K}$, by passing from $\mathcal{D}(x, q)$ to $\operatorname{cl} \mathcal{D}(x, q)$, we do not change the auxiliary dual value function.

Lemma 3.16. Let the conditions of Theorem 3.1 hold and $(x, q) \in \mathcal{K}$. Then, for every $z>0$, we have

$$
-\infty<\inf _{Y \in \operatorname{cl} \mathcal{D}(x, q)} \mathbb{E}\left[\int_{0}^{T} V\left(s, z Y_{s}\right) d \kappa_{s}\right]=\inf _{Y \in \mathcal{D}(x, q)} \mathbb{E}\left[\int_{0}^{T} V\left(s, z Y_{s}\right) d \kappa_{s}\right]<\infty .
$$

Proof. Finiteness from above follows from Lemma 3.14. To show finiteness of both infima in (38) from below, by Lemma 3.15 we deduce the existence of $c \in \mathcal{A}(x, q)$, such that

$$
\mathbb{E}\left[\int_{0}^{T} U\left(s, c_{s}\right) d \kappa_{s}\right]>-\infty .
$$

Let $Y \in \operatorname{cl} \mathcal{D}(x, q)$ and let $Y^{n} \in \mathcal{Y}\left(y^{n}, r^{n}\right), n \geq 1$, be a sequence in $\mathcal{D}(x, q)$ that converges to $Y$ in $\mathbb{L}^{0}(d \kappa \times \mathbb{P})$. By Fatou's lemma, Proposition 3.4, and the definition of the set $\mathcal{B}(x, q)$ in $(29)$, we get

$$
\mathbb{E}\left[\int_{0}^{T} Y_{s} c_{s} d \kappa_{s}\right] \leq \liminf _{n \rightarrow \infty} \mathbb{E}\left[\int_{0}^{T} Y_{s}^{n} c_{s} d \kappa_{s}\right] \leq \sup _{n \geq 1}\left(x y^{n}+\int_{0}^{T} r_{s}^{n} q_{s} d K_{s}\right) \leq 1 .
$$

Therefore, we obtain

$$
\begin{aligned}
\mathbb{E}\left[\int_{0}^{T} U\left(s, c_{s}\right) d \kappa_{s}\right] & \leq \mathbb{E}\left[\int_{0}^{T} U\left(s, c_{s}\right) d \kappa_{s}\right]+1-\mathbb{E}\left[\int_{0}^{T} Y_{s} c_{s} d \kappa_{s}\right] \\
& \leq \mathbb{E}\left[\int_{0}^{T} V\left(s, Y_{s}\right) d \kappa_{s}\right]+1,
\end{aligned}
$$


which together with (39) implies finiteness of both infima in (38) from below.

Let us show equality of two infima in (38). It follows from Lemma 3.14 that for every $z>0$ there exists a process $Y \in z \mathcal{D}(x, q)$, such that

$$
\mathbb{E}\left[\int_{0}^{T} V\left(s, Y_{s}\right) d \kappa_{s}\right]<\infty .
$$

Let us fix $z>0$ and let $\bar{Y} \in \operatorname{cl} \mathcal{D}(x, q)$. Also, let $\left(Y^{n}\right)_{n \in \mathbb{N}}$ be a sequence in $\mathcal{D}(x, q)$ that converges to $\bar{Y}(d \kappa \times \mathbb{P})$-a.e. Let us fix $\delta>0$, then by Lemma 3.11, there exists $Z^{\prime} \in \mathcal{Z}^{\prime}$, such that

$$
\mathbb{E}\left[\int_{0}^{T} V\left(t, \delta \bar{y}(x, q) Z_{t}^{\prime}\right) d \kappa_{t}\right]<\infty
$$

where $\bar{y}(x, q)$ is defined in (32). Note that $\bar{y}(x, q) Z^{\prime} \in \mathcal{D}(x, q)$ by Lemma 3.14 (see (33) $)$. Therefore, using Fatou's lemma and monotonicity of $V$ in the spatial variable, we obtain

$$
\begin{aligned}
\inf _{Y \in \mathcal{D}(x, q)} \mathbb{E}\left[\int_{0}^{T} V\left(t,(z+\delta) Y_{s}\right) d \kappa_{s}\right] & \leq \limsup _{n \rightarrow \infty} \mathbb{E}\left[\int_{0}^{T} V\left(t, z Y_{s}^{n}+\delta \bar{y}(x, q) Z_{s}^{\prime}\right) d \kappa_{s}\right] \\
& \leq \mathbb{E}\left[\int_{0}^{T} V\left(t, z \bar{Y}_{s}+\delta \bar{y}(x, q) Z_{s}^{\prime}\right) d \kappa_{s}\right] \\
& \leq \mathbb{E}\left[\int_{0}^{T} V\left(t, z \bar{Y}_{s}\right) d \kappa_{s}\right] .
\end{aligned}
$$

Taking the infimum over $Y \in \operatorname{cl} \mathcal{D}(x, y)$, we deduce that

$$
\inf _{Y \in \mathcal{D}(x, q)} \mathbb{E}\left[\int_{0}^{T} V\left(t,(z+\delta) Y_{s}\right) d \kappa_{s}\right] \leq \inf _{Y \in \operatorname{cl} \mathcal{D}(x, y)} \mathbb{E}\left[\int_{0}^{T} V\left(t, z Y_{s}\right) d \kappa_{s}\right] .
$$

Let us consider

$$
\phi(z):=\inf _{Y \in \mathcal{D}(x, q)} \mathbb{E}\left[\int_{0}^{T} V\left(t, z Y_{s}\right) d \kappa_{s}\right], \quad z>0 .
$$

By the first part of the proof (finiteness of both infima), $\phi$ is finite-valued on $(0, \infty)$. Convexity of $V$ in the spatial variable implies that $\phi$ is also convex. Therefore, $\phi$ is continuous. As (40) holds for every $\delta>0$, by taking the limit as $\delta \downarrow 0$ in (40), we conclude that both infima in (38) are equal. This completes the proof of the lemma.

Let us define

$$
\mathcal{E}:=\{(y, r) \in \mathcal{L}: v(y, r)<\infty\}
$$

Lemma 3.17. Under the conditions of Theorem 3.1, for every $(y, r) \in \mathcal{L}$, we have

$$
v(y, r)>-\infty
$$

Therefore, $v>-\infty$ on $\mathbb{R} \times \mathbb{L}^{1}(d K)$. The set $\mathcal{E}$ is a nonempty convex subset of $\mathcal{L}$, whose closure in $\mathbb{R} \times \mathbb{L}^{1}(d K)$ equals to $\mathcal{L}$, and such that

$$
\mathcal{E}=\bigcup_{\lambda \geq 1} \lambda \mathcal{E}
$$


Proof. Let us fix $(y, r) \in \mathcal{L}$, then finiteness of $v(y, r)$ from below follows from (finValue) and Lemma 3.12, To establish the properties of $\mathcal{E}$, we observe that the convexity of $\mathcal{E}$ and (42) follow from convexity and monotonicity of $V$, respectively.

In remains to show that the closure of $\mathcal{E}$ in $\mathbb{R} \times \mathbb{L}^{1}(d K)$ contains the origin. In (29), let us consider $(x, q)=(1,0) \in \mathcal{K}$. In this case, we have

$$
\mathcal{D}(1,0)=\bigcup_{(y, r) \in \mathcal{L}: y \leq 1} \mathcal{Y}(y, r) \subseteq \mathcal{Y}(1),
$$

where the last inclusion follows from the very definition of $\mathcal{Y}(y, r)$ 's in (7). As, by (6) $\mathcal{Y}(1)$ is closed in $\mathbb{L}^{0}(d \kappa \times \mathbb{P})$ and $\mathcal{D}(1,0) \subseteq \mathcal{Y}(1)$, we deduce that

$$
\operatorname{cl} \mathcal{D}(1,0) \subseteq \mathcal{Y}(1)
$$

By Lemma 3.14, $\mathcal{Z}^{\prime} \subset \mathcal{D}(1,0)$, as $\bar{y}(1,0)=1$. Therefore, by the bipolar theorem of Brannath and Schachermayer, [BS99, Theorem 1.3], we get

$$
\left(\mathcal{Z}^{\prime}\right)^{o o} \subseteq \operatorname{cl} \mathcal{D}(1,0)
$$

On the other hand, Lemma 3.11 asserts that

$$
\left(\mathcal{Z}^{\prime}\right)^{o o}=\mathcal{Y}(1)
$$

Combining (43), (44), and (45), we conclude

$$
\operatorname{cl} \mathcal{D}(1,0)=\mathcal{Y}(1)
$$

Therefore, the sets $\operatorname{cl} \mathcal{D}(1,0)=\mathcal{Y}(1)$ and $\mathcal{A}(1,0)$ satisfy the precise technical assumptions of [Mos15, Theorem 3.2], which, for every $x>0$, grants the existence of $\widehat{c}(x) \in \mathcal{A}(x, 0)$, the unique maximizer to $w(x)$, where $w$ is defined in (10). For every $x>0$, we set

$$
Y .(x):=U^{\prime}(\cdot, \widehat{c} .(x)), \quad(d \kappa \times \mathbb{P})-\text { a.e. }
$$

By [Mos15, Theorem 3.2], for every $x>0, Y(x)$ satisfies

$$
Y(x) \in w^{\prime}(x) \operatorname{cl} \mathcal{D}(1,0) \quad \text { and } \quad \mathbb{E}\left[\int_{0}^{T} V\left(t, Y_{t}(x)\right) d \kappa_{t}\right]<\infty, \quad x>0,
$$

where by [Mos15, Theorem 3.2], $w$ is a strictly concave, differentiable function on $(0, \infty)$ that satisfies the Inada conditions. Therefore, as $w^{\prime}(x)$ can be arbitrary close to 0 (by taking $x$ large enough an by using the Inada conditions) and by Lemmas 3.14 and 3.16, we conclude that the closure of $\mathcal{E}$ in $\mathbb{R} \times \mathbb{L}^{1}(d K)$ contains origin.

In order to prove that the closure of $\mathcal{E}$ in $\mathbb{R} \times \mathbb{L}^{1}(d K)$ equals to $\mathcal{L}$, let $(y, r) \in \mathcal{L} \backslash(0,0)$ be fixed. Let us take $\varepsilon>0$. We want to find $(\tilde{y}, \tilde{r})$, such that

$$
|\tilde{y}-y|+\|\tilde{r}-r\|_{\mathbb{L}^{1}(d K)}<\varepsilon,
$$


and

$$
(\tilde{y}, \tilde{r}) \in \mathcal{E}
$$

As the closure of $\mathcal{E}$ in $\mathbb{R} \times \mathbb{L}^{1}(d K)$ contains origin, we can pick $\left(y^{0}, r^{0}\right) \in \mathcal{E}$, such that

$$
\left|y^{0}\right|+\left\|r^{0}\right\|_{\mathbb{L}^{1}(d K)} \leq \varepsilon / 3
$$

and $Y \in \mathcal{Y}\left(y^{0}, r^{0}\right)$, such that

$$
\mathbb{E}\left[\int_{0}^{T} V\left(t, Y_{t}\right) d \kappa_{t}\right]<\infty
$$

Let us fix $\alpha>1$, such that

$$
\frac{|y|+\|r\|_{\mathbb{L}^{1}(d K)}}{\alpha} \leq \varepsilon / 3
$$

and set $\varepsilon^{\prime}:=\frac{1}{\alpha} \in(0,1)$. By (42) $),\left(\alpha y^{0}, \alpha r^{0}\right) \in \mathcal{E}$. Let

$$
\tilde{y}:=\left(1-\varepsilon^{\prime}\right) y+\varepsilon^{\prime} \alpha y^{0}, \quad \tilde{r}:=\left(1-\varepsilon^{\prime}\right) r+\varepsilon^{\prime} \alpha r^{0} .
$$

Then

$$
\begin{aligned}
|y-\tilde{y}|+\|r-\tilde{r}\|_{\mathbb{L}^{1}(d K)} & =\varepsilon^{\prime} \alpha\left|\frac{y}{\alpha}-y^{0}\right|+\varepsilon^{\prime} \alpha|| \frac{r}{\alpha}-r^{0} \|_{\mathbb{L}^{1}(d K)} \\
& \leq \frac{|y|+|| r \|_{\mathbb{L}^{1}(d K)}}{\alpha}+\left|y^{0}\right|+\left\|r^{0}\right\|_{\mathbb{L}^{1}(d K)} \\
& \leq \frac{2 \varepsilon}{3},
\end{aligned}
$$

where in the last inequality we have used (48) and (50). Thus $(\tilde{y}, \tilde{r})$ satisfies (46). Further, as $0 \in \mathcal{Y}(y, r)$, by convexity of $\mathcal{L}$ and using Proposition 3.4, we get

$$
Y=\left(1-\varepsilon^{\prime}\right) 0+\varepsilon^{\prime} \alpha Y \in \mathcal{Y}(\tilde{y}, \tilde{r}),
$$

which by (49) implies (47). This completes the proof of the lemma.

\subsection{Existence and uniqueness of solutions to (2) and (8); semicontinuity and} biconjugacy of $u$ and $v$.

Lemma 3.18. Under the conditions of Theorem 3.1, the value function $v$ is convex, proper, and lower semicontinuous with respect to the topology of $\mathbb{R} \times \mathbb{L}^{1}(d K)$. For every $(y, r) \in \mathcal{E}$, there exists a unique solution to (8). Likewise, $u$ is concave, proper, and upper semicontinuous with respect to the strong topology of $\mathbb{R} \times \mathbb{L}^{\infty}(d K)$. For every $(x, q) \in\{u>-\infty\}$ there exists a unique solution to (2).

Proof. Let $\left(y^{n}, r^{n}\right)_{n \in \mathbb{N}}$ be a sequence in $\mathcal{L}$ that converges to $(y, r)$ in $\mathbb{R} \times \mathbb{L}^{1}(d K)$. Passing if necessary to a subsequence, we will assume that

$$
\lim _{n \rightarrow \infty} v\left(y^{n}, r^{n}\right)=\liminf _{n \rightarrow \infty} v\left(y^{n}, r^{n}\right) .
$$


Let $Y^{n} \in \mathcal{Y}\left(y^{n}, r^{n}\right), n \in \mathbb{N}$, be such that

$$
\mathbb{E}\left[\int_{0}^{T} V\left(t, Y_{t}^{n}\right) d \kappa_{t}\right] \leq v\left(y^{n}, r^{n}\right)+\frac{1}{n}, \quad n \in \mathbb{N} .
$$

By passing to convex combinations and applying Komlos'-type lemma, see e.g. [DS94, Lemma A1.1], we may suppose that $\widetilde{Y}^{n} \in \operatorname{conv}\left(Y^{n}, Y^{n+1}, \ldots\right), n \in \mathbb{N}$, converges $(d \kappa \times \mathbb{P})$ a.e. to some $\widehat{Y}$.

For every $(x, q) \in \mathcal{K}$ and $c \in \mathcal{A}(x, q)$, by Fatou's lemma, we have

$$
\mathbb{E}\left[\int_{0}^{T} c_{t} \widehat{Y}_{t} d \kappa_{t}\right] \leq \liminf _{n \rightarrow \infty} \mathbb{E}\left[\int_{0}^{T} c_{t} \widetilde{Y}_{t}^{n} d \kappa_{t}\right] \leq x y+\int_{0}^{T} q_{s} r_{s} d K_{s} .
$$

Therefore, by Proposition 3.4, $\widehat{Y} \in \mathcal{Y}(y, r)$. With $\bar{y}:=\sup _{n \geq 1} y^{n}$, we have $\left(\widetilde{Y}^{n}\right)_{n \in \mathbb{N}} \subseteq \mathcal{Y}(\bar{y})$. Therefore, by [Mos15, Lemma 3.5], we deduce that $V^{-}\left(t, \widetilde{Y}_{t}^{n}\right), n \in \mathbb{N}$, is a uniformly integrable sequence. Combining uniform integrability with the convexity of $V$ in the spatial variable, we get

$$
\begin{gathered}
v(y, r) \leq \mathbb{E}\left[\int_{0}^{T} V\left(t, \widehat{Y}_{t}\right) d \kappa_{t}\right] \leq \liminf _{n \rightarrow \infty} \mathbb{E}\left[\int_{0}^{T} V\left(t, \widetilde{Y}_{t}^{n}\right) d \kappa_{t}\right] \\
\leq \liminf _{n \rightarrow \infty} \mathbb{E}\left[\int_{0}^{T} V\left(t, Y_{t}^{n}\right) d \kappa_{t}\right]=\liminf _{n \rightarrow \infty} v\left(y^{n}, r^{n}\right),
\end{gathered}
$$

where in the last equality we have used (51) and (52). Since $\left(y^{n}, q^{n}\right)$ was an arbitrary sequence that converges to $(y, r)$, lower semicontinuity of $v$ in strong topology of $\mathbb{R} \times$ $\mathbb{L}^{1}(d K)$ follows. Since $\mathcal{L}$ is closed and $v=\infty$ outside of $\mathcal{L}$, we deduce that $v$ is lower semicontinuous on $\mathbb{R} \times \mathbb{L}^{1}(d K)$. The function $v$ is proper by Lemma 3.17. Note that (53) also implies that $\mathcal{E}$ defined in (41) is $\mathbb{R} \times \mathbb{L}^{1}(d K)$-norm closed. For $(y, r) \in \mathcal{E}$, by taking $\left(y^{n}, r^{n}\right)=(y, r), n \in \mathbb{N}$, we deduce the existence of a minimizer to (8) . Strict convexity of $V$ results in the uniqueness of the minimizer to (8) . Convexity of $v$ follows. Upper semicontinuity of $u$ with respect to the norm-topology of $\mathbb{R} \times \mathbb{L}^{\infty}(d K)$ can be proven similarly, first proving semi-continuity on $\mathcal{K}$ by a Fatou-type argument, then using the closedness of $\mathcal{K}$ and the definition of $u$ outside it.

Corollary 3.19. Under the conditions of Theorem [3.1, $-u$ and $v$ are also lower semicontinuous with respect to the weak topologies $\sigma\left(\mathbb{R} \times \mathbb{L}^{\infty}(d K),\left(\mathbb{R} \times \mathbb{L}^{\infty}\right)^{*}(d K)\right)$ and $\sigma(\mathbb{R} \times$ $\left.\mathbb{L}^{1}(d K), \mathbb{R} \times \mathbb{L}^{\infty}(d K)\right)$, respectively.

Proof. The assertions of the corollary is a consequence of [BP12, Proposition 2.2.10], see also [ET76, Corollary I.2.2].

We recall that $\operatorname{cl} \mathcal{D}(x, q)$ denotes the closure of $\mathcal{D}(x, q)$ in $\mathbb{L}^{0}(d \kappa \times \mathbb{P})$.

Lemma 3.20. Under the conditions of Theorem [3.1, for every $(x, q)$ in $\mathcal{K}$, and a nonnegative optional process $c$, we have

$$
c \in \mathcal{A}(x, q) \quad \text { if and only if } \quad \mathbb{E}\left[\int_{0}^{T} c_{s} Y_{s} d \kappa_{s}\right] \leq 1 \quad \text { for every } Y \in \operatorname{cl} \mathcal{D}(x, q) .
$$


Proof. Let $(x, q)$ in $\dot{\mathcal{K}}, c$ is a nonnegative optional process such that

$$
\mathbb{E}\left[\int_{0}^{T} c_{s} Y_{s} d \kappa_{s}\right] \leq 1 \quad \text { for every } \quad Y \in \operatorname{cl} \mathcal{D}(x, q) .
$$

Consider arbitrary $Z \in \mathcal{Z}^{\prime}$ and $\Lambda \in \Upsilon$. Let the corresponding $r$ be given by (16) and we set

$$
y^{\prime}:=x+\int_{0}^{T} q_{s} r_{s} d K_{s}
$$

If $y^{\prime}=0$, then $y \Lambda Z \in \mathcal{D}(x, q)$ for every $y>0$. Thus, by (54), we obtain that $\mathbb{E}\left[\int_{0}^{T} c_{s} y \Lambda_{s} Z_{s} d \kappa_{s}\right] \leq 1$. Taking the limit as $y \rightarrow \infty$, we get

$$
\mathbb{E}\left[\int_{0}^{T} c_{s} \Lambda_{s} Z_{s} d \kappa_{s}\right]=0=x+\int_{0}^{T} q_{s} r_{s} d K_{s}=x+\mathbb{E}\left[\int_{0}^{T} q_{s} e_{s} \Lambda_{s} Z_{s} d \kappa_{s}\right]
$$

where in the last equality we have used (16).

If $y^{\prime}>0$, then $\frac{1}{y^{\prime}} \Lambda Z \in \mathcal{D}(x, q)$ and thus by (54), we obtain

$$
\mathbb{E}\left[\int_{0}^{T} c_{s} \frac{1}{y^{\prime}} \Lambda_{s} Z_{s} d \kappa_{s}\right] \leq 1=\frac{x+\int_{0}^{T} q_{s} r_{s} d K_{s}}{y^{\prime}}=\frac{1}{y^{\prime}}\left(x+\mathbb{E}\left[\int_{0}^{T} q_{s} e_{s} \Lambda_{s} Z_{s} d \kappa_{s}\right]\right)
$$

where in the last equality, we have used (16) again. Consequently, we deduce

$$
\mathbb{E}\left[\int_{0}^{T} c_{s} \Lambda_{s} Z_{s} d \kappa_{s}\right] \leq x+\mathbb{E}\left[\int_{0}^{T} q_{s} e_{s} \Lambda_{s} Z_{s} d \kappa_{s}\right]
$$

which together with (55), by Lemma 3.10, imply that $c \in \mathcal{A}(x, q)$.

Conversely, let $(x, q) \in \dot{\mathcal{K}}, c \in \mathcal{A}(x, q)$ and $Y \in \operatorname{cl} \mathcal{D}(x, q)$. Then there exists a sequence $Y^{n} \in \mathcal{Y}\left(y^{n}, r^{n}\right)$ convergent to $Y,(d \kappa \times \mathbb{P})$-a.e., where $\left(y^{n}, r^{n}\right) \in \mathcal{B}(x, q)$. As,

$$
\mathbb{E}\left[\int_{0}^{T} c_{s} Y_{s}^{n} d \kappa_{s}\right] \leq 1, \quad n \in \mathbb{N}
$$

by Fatou's lemma, we get

$$
\mathbb{E}\left[\int_{0}^{T} c_{s} Y_{s} d \kappa_{s}\right] \leq \liminf _{n \rightarrow \infty} \mathbb{E}\left[\int_{0}^{T} c_{s} Y_{s}^{n} d \kappa_{s}\right] \leq 1
$$

This completes the proof of the lemma.

Lemma 3.21. Under the conditions of Theorem 3.1, for every $(x, q)$ in $\dot{\mathcal{K}}$, we have

$$
u(x, q)=\inf _{(y, r) \in \mathcal{L}}\left(v(y, r)+x y+\int_{0}^{T} r_{s} q_{s} d K_{s}\right) .
$$

Proof. Let us fix $(x, q) \in \dot{\mathcal{K}}$. By Lemma 3.14, $\mathcal{A}(x, q)$ and $\operatorname{cl} \mathcal{D}(x, q)$ contain strictly positive elements. Therefore, using Lemma 3.20 we deduce that the sets $\mathcal{A}(x, q)$ and $\operatorname{cl} \mathcal{D}(x, q)$ 
satisfy the assumptions of [Mos15, Theorem 3.2]. From this theorem, Lemma 3.16, and the definition of the set $\mathcal{B}(x, q)$, we get

$$
\begin{aligned}
u(x, q) & =\inf _{z>0}\left(\inf _{Y \in \operatorname{cl} \mathcal{D}(x, q)} \mathbb{E}\left[\int_{0}^{T} V\left(t, z Y_{s}\right) d \kappa_{s}\right]+z\right) \\
& =\inf _{z>0}\left(\inf _{Y \in \mathcal{D}(x, q)} \mathbb{E}\left[\int_{0}^{T} V\left(t, z Y_{s}\right) d \kappa_{s}\right]+z\right) \\
& =\inf _{z>0}\left(\inf _{(y, r) \in z \mathcal{B}(x, q)} v(y, r)+z\right) \\
& \geq \inf _{(y, r) \in \mathcal{L}}\left(v(y, r)+x y+\int_{0}^{T} q_{s} r_{s} d K_{s}\right) .
\end{aligned}
$$

Combining this with the conclusion of Lemma 3.12, we deduce that (56) holds for every $(x, q) \in \stackrel{\circ}{\mathcal{K}}$.

Before proving the biconjugacy relations of item (iii), Theorem 3.1, we need a preliminary lemma. Essentially following the notations in [ET76], we define

$$
\begin{aligned}
& \text { (57) } v^{*}(x, q):=\inf _{(y, r) \in \mathbb{R} \times \mathbb{L}^{1}(d K)}\left(v(y, r)+x y+\int_{0}^{T} q_{s} r_{s} d K_{s}\right), \quad(x, q) \in \mathbb{R} \times \mathbb{L}^{\infty}(d K) . \\
& \text { (58) } \quad v^{* *}(y, r):=\sup _{(x, q) \in \mathbb{R} \times \mathbb{L}^{\infty}(d K)}\left(v^{*}(x, q)-x y-\int_{0}^{T} q_{s} r_{s} d K_{s}\right), \quad(y, r) \in \mathbb{R} \times \mathbb{L}^{1}(d K) .
\end{aligned}
$$

Remark 3.22. In [ET76], conjugate convex functions are considered on general spaces $V$ and $V^{*}$ supplied with $\sigma\left(V, V^{*}\right)$ and $\sigma\left(V^{*}, V\right)$ topologies, which in our case are $V=$ $\mathbb{R} \times \mathbb{L}^{1}(d K), V^{*}=\mathbb{R} \times \mathbb{L}^{\infty}(d K)$. Thus, the starting point of our analysis is $v$, not $u$. We remind the reader we have already proved that the dual value function $v$ is convex, proper and lower-semicontinuous on the space $V=\mathbb{R} \times \mathbb{L}^{1}(d K)$.

Lemma 3.23. Under the conditions of Theorem 3.1, we have

$$
\begin{gathered}
v^{* *}=v, \\
v^{*}(x, q)=-\infty, \quad \text { for every }(x, q) \in \mathbb{R} \times \mathbb{L}^{\infty}(d K) \backslash \mathcal{K} .
\end{gathered}
$$

Proof. To show (59), we observe that by Lemma 3.18, $v$ is lower semicontinuous in the $\mathbb{R} \times \mathbb{L}^{1}(d K)$-norm topology (and therefore, by [ET76, Corollary I.2.2], also in the weak topology $\left.\sigma\left(\mathbb{R} \times \mathbb{L}^{1}(d K), \mathbb{R} \times \mathbb{L}^{\infty}(d K)\right)\right)$. As a result, by [ET76, Proposition I.4.1], we get (159).

The proof of (60) will be done in several steps.

Step 1. Let $(x, q) \in \mathbb{R} \times \mathbb{L}^{\infty}(d K) \backslash \mathcal{K}$. According to Proposition 3.4 there exists $(y, r) \in$ $\mathcal{L}$, such that

$$
C:=x y+\int_{0}^{T} q_{s} r_{s} d K_{s}<0 .
$$


Therefore, as $\mathcal{L}$ is a cone, for every $a>0,(a y, a r) \in \mathcal{L}$, and we have

$$
x a y+\int_{0}^{T} q_{s} a r_{s} d K_{s}=a C<0 .
$$

Note that $0 \in \mathcal{Y}(a y, a r), a>0$.

Step 2. Let us consider $Z \in \mathcal{Z}^{\prime}$, such that

$$
\mathbb{E}\left[\int_{0}^{T} V\left(t, \frac{1}{2} Z_{t}\right) d \kappa_{t}\right]<\infty .
$$

The existence of such a $Z$ is granted by Lemma 3.11. Further, by Corollary 3.9, there exists $\rho \in \mathbb{L}^{1}(d K)$, such that $(1, \rho) \in \mathcal{L}, Z \in \mathcal{Y}(1, \rho)$, and

$$
\int_{0}^{t} \rho_{s} d K_{s}=\mathbb{E}\left[\int_{0}^{t} Z_{s} e_{s} d \kappa_{s}\right], \quad t \in[0, T] .
$$

Let us set

$$
D:=x+\int_{0}^{T} q_{s} \rho_{s} d K_{s} \in \mathbb{R} .
$$

Step 3. In (61), let us pick

$$
a=\frac{|D|+1}{-C}>0
$$

Then, we have

$$
a C+D=-|D|+D-1<0 .
$$

Step 4. Let us define

$$
Y:=\frac{1}{2} Z, \quad y^{\prime}:=\frac{1}{2} a y+\frac{1}{2}, \quad \text { and } \quad r^{\prime}:=\frac{1}{2} a r+\frac{1}{2} \rho .
$$

Then by (62), we obtain

$$
\mathbb{E}\left[\int_{0}^{T} V\left(t, Y_{t}\right) d \kappa_{t}\right]<\infty
$$

As $Z \in \mathcal{Y}(1, \rho)$ and $0 \in \mathcal{Y}($ ay, ar $)$, by convexity of $\mathcal{L}$ and Proposition 3.4, we have

$$
Y \in \mathcal{Y}\left(y^{\prime}, r^{\prime}\right)
$$

where $y^{\prime}$ and $r^{\prime}$ are defined in (64). Now, it follows from (65) and (666) that $\left(y^{\prime}, r^{\prime}\right) \in \mathcal{E}$ (where $\mathcal{E}$ is defined in (41)). Therefore, we obtain

$$
\begin{aligned}
& 2\left(x y^{\prime}+\int_{0}^{T} q_{s} r_{s}^{\prime} d K_{s}\right)=(a y+1) x+\int_{0}^{T}\left(a r_{s}+\rho_{s}\right) q_{s} d K_{s} \\
= & a\left(x y+\int_{0}^{T} q_{s} r_{s} d K_{s}\right)+x+\int_{0}^{T} q_{s} \rho_{s} d K_{s}=a C+D<0,
\end{aligned}
$$

where the last inequality follows from (63). To recapitulate, we have shown the existence of $\left(y^{\prime}, r^{\prime}\right)$, such that

$$
\left(y^{\prime}, r^{\prime}\right) \in \mathcal{E} \quad \text { and } \quad x y^{\prime}+\int_{0}^{T} q_{s} r_{s}^{\prime} d K_{s}<0 .
$$


Step 6. For $y^{\prime}$ and $r^{\prime}$ defined in (64), as $v\left(y^{\prime}, r^{\prime}\right)<\infty$ and $x y^{\prime}+\int_{0}^{T} q_{s} r_{s}^{\prime} d K_{s}<0$ by (67), from the monotonicity of $V$, we get

$$
\infty>v\left(y^{\prime}, r^{\prime}\right) \geq v\left(\lambda y^{\prime}, \lambda r^{\prime}\right), \quad \lambda \geq 1 .
$$

As $\bigcup_{\lambda \geq 1}\left(\lambda y^{\prime}, \lambda r^{\prime}\right) \subset \mathcal{L}$, we conclude via (67) that

$$
v^{*}(x, q) \leq \lim _{\lambda \rightarrow \infty}\left(v\left(\lambda y^{\prime}, \lambda r^{\prime}\right)+\lambda\left(x y^{\prime}+\int_{0}^{T} q_{s} r_{s}^{\prime} d K_{s}\right)\right)=-\infty .
$$

Therefore, (60) holds. This completes the proof of the lemma.

Lemma 3.24. Under the conditions of Theorem 3.1, we have

$$
\begin{aligned}
& v(y, r)=\sup _{(x, q) \in \mathcal{K}}\left(u(x, q)-x y-\int_{0}^{T} r_{s} q_{s} d K_{s}\right), \quad(y, r) \in \mathcal{L}, \\
& u(x, q)=\inf _{(y, r) \in \mathcal{L}}\left(v(y, r)+x y+\int_{0}^{T} q_{s} r_{s} d K_{s}\right), \quad(x, q) \in \mathcal{K} .
\end{aligned}
$$

Proof. Lemma 3.21 and (9) imply that on $\mathcal{K}$, for $v^{*}$ defined in (57), we have

$$
v^{*}=u \text {. }
$$

By Lemma 3.23, $v^{*}=-\infty$ on $\mathbb{R} \times \mathbb{L}^{\infty}(d K) \backslash \mathcal{K}$. From [ET76, Definition I.4.1] and Lemma 3.18, respectively, we deduce that both $v^{*}$ and $u$ are upper semicontinuous in the topology of $\mathbb{R} \times \mathbb{L}^{\infty}(d K)$. Consequently, from (70), using [ET76, Corollary I.2.1], we get

$$
v^{*}=u, \quad \text { on } \quad \mathbb{R} \times \mathbb{L}^{\infty}(d K) .
$$

As a result, with $v^{* *}$ being defined in (58), for every $(y, r) \in \mathbb{R} \times \mathbb{L}^{1}(d K)$, we obtain

$$
u^{*}(y, r):=\sup _{(x, q) \in \mathbb{R} \times \mathbb{L}^{\infty}(d K)}\left(u(x, q)-x y-\int_{0}^{T} q_{s} r_{s} d K_{s}\right)=v^{* *}(y, r) .
$$

Therefore, from (159) in Lemma 3.23 and (172), we get

$$
v=u^{*}, \quad \text { on } \quad \mathbb{R} \times \mathbb{L}^{1}(d K) .
$$

As a result, applying Lemma 3.23 again and since $u=-\infty$ outside of $\mathcal{K}$ by (9), we deduce

$$
\begin{aligned}
v(y, r) & =\sup _{(x, q) \in \mathbb{R} \times \mathbb{L}^{\infty}(d K)}\left(u(x, q)-x y-\int_{0}^{T} r_{s} q_{s} d K_{s}\right) \\
& =\sup _{(x, q) \in \mathcal{K}}\left(u(x, q)-x y-\int_{0}^{T} r_{s} q_{s} d K_{s}\right), \quad(y, r) \in \mathcal{L},
\end{aligned}
$$

Thus, (68) holds.

In turn, from (71) using (9), we conclude that 


$$
\begin{aligned}
u(x, q) & =\inf _{(y, r) \in \mathbb{R} \times \mathbb{L}^{1}(d K)}\left(v(y, r)+x y+\int_{0}^{T} q_{s} r_{s} d K_{s}\right), \\
& =\inf _{(y, r) \in \mathcal{L}}\left(v(y, r)+x y+\int_{0}^{T} q_{s} r_{s} d K_{s}\right), \quad(x, q) \in \mathcal{K},
\end{aligned}
$$

which proves (69) and extends the assertion of Lemma 3.21 to the boundary of $\mathcal{K}$.

Proof of Theorem 3.1. The assertions of item $(i)$ follow from Lemmas 3.15 and 3.17, item (ii) results from Lemma 3.18, whereas the validity of item (iii) come from Lemma 3.24. This completes the proof of the theorem.

\section{SubdiffEREntiability of $u$}

In order to establish subdifferentiability of $u$, we need to strengthen Assumption 2.5 and to impose the following condition.

Assumption 4.1. There exists an a.s. bounded away from 0 and $\infty$ process $\varphi$, such that

$$
d \kappa(\omega)=\varphi d K, \quad \text { for } \quad \mathbb{P}-\text { a.e. } \quad \omega \in \Omega .
$$

Let

$$
\mathcal{P}:=\{\rho:(1, \rho) \in \mathcal{L}\}
$$

Remark 4.2. $\mathcal{P}$ defined in (73) needs to be uniformly integrable with respect to the measure $d K$ in order for the proof of subdifferentiability of $u$ to go through. Assumption 2.5 through Lemma 3.2 only implies that $\mathcal{P}$ is $\mathbb{L}^{1}$ bounded. A stronger condition on the stochastic clock and income stream is, therefore, needed to obtain uniform integrability.

The following theorem characterizes subdifferentiability of $u$ over $\mathcal{K}$, where we are looking for an $\mathbb{R} \times \mathbb{L}^{1}(d K)$-valued subgradient. Under our assumptions, we can find elements of the sub gradient which both belong to the effective domain of $v, \mathcal{E}$, and are bounded, i.e. in $\mathbb{R} \times \mathbb{L}^{\infty}(d K)$.

Theorem 4.3. Let the conditions of Theorem [3.1 and Assumption 4.1 hold. Then for every $(x, q) \in \mathcal{K}$, the subdifferential of $u$ at $(x, q)$ is a nonempty and contains an element of $\mathcal{E}$, i.e.,

$$
\partial u(x, q) \cap \mathcal{E} \neq \emptyset .
$$

Moreover, for $(x, q) \in \stackrel{\mathcal{K}}{ }$ and $(y, r) \in \mathcal{L},(y, r) \in \partial u(x, q)$ if and only if the following conditions hold:

$$
|v(y, r)|<\infty
$$


thus, $(y, r) \in \mathcal{E}$,

$$
\begin{gathered}
\mathbb{E}\left[\int_{0}^{T} \widehat{Y}_{t}(y, r) \widehat{c}_{t}(x, q) d \kappa_{t}\right]=x y+\int_{0}^{T} q_{s} r_{s} d K_{s}, \\
\widehat{Y}_{t}(y, r)=U^{\prime}\left(t, \widehat{c}_{t}(x, q)\right), \quad(d \kappa \times \mathbb{P})-\text { a.e. },
\end{gathered}
$$

where $\widehat{c}(x, q)$ and $\widehat{Y}(y, r)$ are the unique optimizers to (21) and (8), respectively.

\subsection{Uniform integrability of $\mathcal{P}$.}

Lemma 4.4. Let the conditions of Theorem 4.3 hold. Then $\mathcal{P}$ is $\mathbb{L}^{\infty}(d K)$-bounded, and, therefore, a uniformly integrable family.

Proof. Step 1. For an arbitrary $q:[0, T] \rightarrow[0,1]$, let us define

$$
\beta(q):=\sup _{\mathbb{Q} \in \mathcal{M}} \mathbb{E}^{\mathbb{Q}}\left[\int_{0}^{T} q(s)\left|e_{s}\right| d \kappa_{s}\right]=\sup _{\mathbb{Q} \in \mathcal{M}, \tau \in \Theta} \mathbb{E}^{\mathbb{Q}}\left[\int_{0}^{\tau} q(s)\left|e_{s}\right| d \kappa_{s}\right] .
$$

Note that by Assumption (2.5), we have

$$
\begin{aligned}
\beta(q) & =\sup _{\mathbb{Q} \in \mathcal{M}} \mathbb{E}^{\mathbb{Q}}\left[\int_{0}^{T} q(s)\left|e_{s}\right| d \kappa_{s}\right] \\
& \leq \sup _{\mathbb{Q} \in \mathcal{M}} \mathbb{E}^{\mathbb{Q}}\left[\int_{0}^{T} q(s) X_{s}^{\prime} \varphi d K_{s}\right] \\
& \leq \int_{0}^{T} q(s) \sup _{\mathbb{Q} \in \mathcal{M}} \mathbb{E}^{\mathbb{Q}}\left[C X_{s}^{\prime}\right] d K_{s} \\
& \leq C X_{0}^{\prime} \int_{0}^{T} q(s) d K_{s},
\end{aligned}
$$

where $C \in \mathbb{R}$ is such that $|\varphi| \leq C$. It follows from [Kra96, Proposition 4.3] and [FK97, Theorem 3.1] that there exists a nonnegative càdlàg process $V=\beta(q)+H \cdot S$, such that

$$
V_{t} \geq \operatorname{ess}_{\mathbb{Q} \in \mathcal{M}, \tau \in \Theta, \tau \geq t} \mathbb{E}^{\mathbb{Q}}\left[\int_{0}^{\tau} q(s)\left|e_{s}\right| d \kappa_{s} \mid \mathcal{F}_{t}\right], \quad t \in[0, T] .
$$

Consequently, $V$ satisfies

$$
V_{\tau} \geq \int_{0}^{\tau} q(s)\left|e_{s}\right| d \kappa_{s} \geq \int_{0}^{\tau} q(s) e_{s} d \kappa_{s}, \quad \mathbb{P}-\text { a.s. }, \quad \tau \in \Theta,
$$

and thus, $(\beta(q),-q) \in \mathcal{K}$. As a result, from the definitions of $\mathcal{L}$ and $\mathcal{P}$, we get

$$
\beta(q) \geq \sup _{\rho \in \mathcal{P}} \int_{0}^{T} q(s) \rho(s) d K_{s} .
$$

One can see that for every $\rho \in \mathcal{P}$, we have $\rho \leq f:=C X_{0}^{\prime}$, $d K$-a.e.

Step 2. For an arbitrary $q:[0, T] \rightarrow[-1,0]$, let us set

$$
\tilde{\beta}(q):=\sup _{\mathbb{Q} \in \mathcal{M}} \mathbb{E}^{\mathbb{Q}}\left[\int_{0}^{T} q(s)\left(-\left|e_{s}\right|\right) d \kappa_{s}\right] .
$$


As in Step 1, we can construct a càdlàg process $\tilde{V}=\tilde{\beta}(q)+H \cdot S$, s.t.

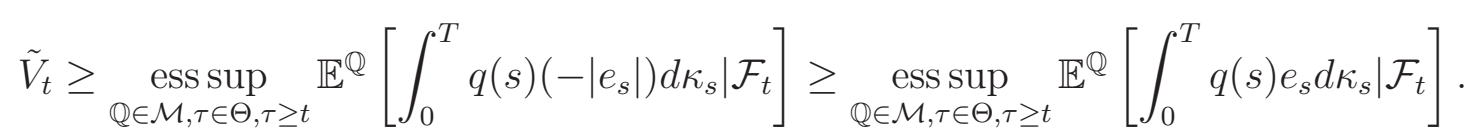

This implies that $(\tilde{\beta}(q),-q) \in \operatorname{cl} \mathcal{K}$. Therefore,

$$
\beta(q) \geq \int_{0}^{T} q(s) \rho(s) d K_{s}, \quad \text { for every } \rho \in \mathcal{P} .
$$

Similarly to Step 1, one can see that $\rho \geq-f$, $d K$-a.e. for every $\rho \in \mathcal{P}$.

Step 3.In view of Steps 1 and 2 , uniform integrability of $\mathcal{P}$ under $d K$ follows from the integrability of $f$ under $d K$.

Lemma 4.5. Let the assumption of Theorem 4.3 hold and $(x, q) \in \mathcal{K}$. Then

$$
\{r:(y, r) \in \mathcal{B}(x, q)\}
$$

is $\mathbb{L}^{\infty}$-bounded, so a uniformly integrable subset of $\mathbb{L}^{1}(d K)$.

Proof. By Lemma 3.13, we deduce the existence of a constant $M>0$, such that

$$
y \leq M, \quad \text { for every }(y, r) \in \mathcal{B}(x, q) .
$$

We conclude that

$$
\{r:(y, r) \in \mathcal{B}(x, q)\} \subseteq \bigcup_{0 \leq \lambda \leq M} \lambda \mathcal{P},
$$

and thus by Lemma 4.4, $\{r:(y, r) \in \mathcal{B}(x, q)\}$ is a uniformly integrable family.

\subsection{Closedness of $\mathcal{D}(x, q)$ for every $(x, q) \in \stackrel{\mathcal{K}}{\text {. }}$}

Lemma 4.6. Under the conditions of Theorem 4.3, for every $(x, q) \in \mathcal{K}$, the set $\mathcal{D}(x, q)$ is closed in $\mathbb{L}^{0}(d \kappa \times \mathbb{P})$.

Proof. Let $(x, q) \in \mathcal{K}_{\mathcal{K}}$ and $Y$ be an arbitrary element of $\operatorname{cl} \mathcal{D}(x, q)$. We claim that there exists $(y, r) \in \mathcal{B}(x, q)$, such that $Y \in \mathcal{Y}(y, r)$. Let $Y^{n} \in \mathcal{Y}\left(y^{n}, r^{n}\right), n \geq 1$, be a sequence in $\mathcal{D}(x, q)$, such that $\lim _{n \rightarrow \infty} Y^{n}=Y,(d \kappa \times \mathbb{P})$-a.e. Since $\left(y^{n}, r^{n}\right)_{n \geq 1} \subset \mathcal{B}(x, q)$, which is bounded in the sense of Lemma 3.13, Komlos' lemma implies the existence of a subsequence of convex combinations $\left(\tilde{y}^{n}, \tilde{r}^{n}\right) \in \operatorname{conv}\left(\left(y^{n}, r^{n}\right),\left(y^{n+1}, r^{n+1}\right), \ldots\right), n \geq 1$, such that $\left(\tilde{y}^{n}\right)_{n \geq 1}$ converges to $y$ and $\left(\tilde{r}^{n}\right)_{n \geq 1}$ converges to $r$, $d K$-a.e. Lemma 4.5 implies that $\left(\tilde{r}^{n}\right)_{n \geq 1}$ is uniformly integrable. Therefore $\left(\tilde{r}^{n}\right)_{n \geq 1}$ converges to $r$ in $\mathbb{L}^{1}(d K)$. Note that the corresponding sequence of convex combinations of $\left(Y^{n}\right)_{n \geq 1},\left(\tilde{Y}^{n}\right)_{n \geq 1}$ converges to $Y,(d \kappa \times$ $\mathbb{P})$-a.e. Then we have

$$
1 \geq \lim _{n \rightarrow \infty}\left(x \tilde{y}^{n}+\int_{0}^{T} q_{s} \tilde{r}_{s}^{n} d K_{s}\right)=x y+\int_{0}^{T} q_{s} r_{s} d K_{s} .
$$

Therefore, $(y, r) \in \mathcal{B}(x, q)$. 
Let us fix an arbitrary $\left(x^{\prime}, q^{\prime}\right) \in \mathcal{K}$ and $c \in \mathcal{A}\left(x^{\prime}, q^{\prime}\right)$. Using Proposition 3.4, Fatou's lemma, and Lemma 4.5, we obtain

$$
\begin{gathered}
0 \leq \mathbb{E}\left[\int_{0}^{T} Y_{s} c_{s} d s\right] \leq \liminf _{n \rightarrow \infty} \mathbb{E}\left[\int_{0}^{T} \tilde{Y}_{s}^{n} c_{s} d s\right] \\
\leq \lim _{n \rightarrow \infty}\left(x^{\prime} \tilde{y}^{n}+\int_{0}^{T} q_{s}^{\prime} \tilde{r}_{s}^{n} d K_{s}\right)=x^{\prime} y+\int_{0}^{T} q_{s}^{\prime} r_{s} d K_{s},
\end{gathered}
$$

where the uniform integrability of $\mathcal{B}(x, q)$ in needed once again in the last equality. From Proposition 3.4, we conclude that that $Y \in \mathcal{Y}(y, r)$.

Proof of Theorem 4.3. Let $(x, q) \in \mathcal{K}, \widehat{c}(x, q)$ be the minimizer to (2), whose existence and uniqueness are established in Lemma 3.18, Let us also set

$$
\widehat{Y}_{t}:=U^{\prime}\left(t, \widehat{c}_{t}(x, q)\right), \quad(t, \omega) \in[0, T] \times \Omega, \quad \text { and } \quad z:=\mathbb{E}\left[\int_{0}^{T} \widehat{c}_{s}(x, q) \widehat{Y}_{s} d \kappa_{s}\right] .
$$

Note that the sets $\mathcal{A}(x, q)$ and $\operatorname{cl} \mathcal{D}(x, q)$ satisfy the conditions of [Mos15, Theorem 3.2], which implies that $\widehat{Y} \in z \operatorname{cl} \mathcal{D}(x, q)$ is the unique solution to the optimization problem

$$
\inf _{Y \in z \operatorname{cl} \mathcal{D}(x, q)} \mathbb{E}\left[\int_{0}^{T} V\left(t, Y_{s}\right) d \kappa_{s}\right]=\mathbb{E}\left[\int_{0}^{T} V\left(t, \widehat{Y}_{s}\right) d \kappa_{s}\right] \in \mathbb{R}
$$

where finiteness follows from Lemma 3.16, Note that by Lemma 4.6, $\widehat{Y} \in \mathcal{Y}(z y, z r)$ for some $(y, r) \in \mathcal{B}(x, q)$. It follows from the definition of $\widehat{Y}$ in (78) that for $\widehat{c}(x, q)$ and $\widehat{Y}$ we have the following relation

$$
U\left(t, \widehat{c}_{t}(x, q)\right)=V\left(t, \widehat{Y}_{t}\right)+\widehat{c}_{t}(x, q) \widehat{Y}_{t}, \quad(t, \omega) \in[0, T] \times \Omega,
$$

which together with Lemma 3.21 implies that

$$
\begin{gathered}
u(x, q)=v(z y, z r)+z\left(x y+\int_{0}^{T} q_{s} r_{s} d K_{s}\right), \\
\widehat{Y}(z y, z r)=\widehat{Y}, \quad(d \kappa \times \mathbb{P})-\text { a.e. }
\end{gathered}
$$

where $\widehat{Y}(z y, z r)$ is the unique minimizer to the dual problem (8). By (79), $(z y, z r) \in \mathcal{E}$. (79) and [ET76, Proposition I.5.1] assert that $(z y, z r) \in \partial u(x, q)$. In particular, we get

$$
\partial u(x, q) \cap \mathcal{E} \neq \emptyset
$$

i.e., (174). Note that even though, e.g., [BP12, Corollary 2.2.38 and Corollary 2.2.44] imply that $\partial u(x, q) \neq \emptyset$, over the interior of the effective domain, its elements are in $\mathbb{R} \times\left(\mathbb{L}^{\infty}\right)^{*}(d K)$. Relation (74) shows that $\partial u(x, q)$ contains at least a bounded element of $\mathcal{E} \subseteq \mathcal{L} \subset \mathbb{R} \times \mathbb{L}^{1}(d K)$.

Let $(x, q) \in \mathcal{K}$ and $(y, r) \in \mathcal{L}$. Suppose that (75), (76), and (777) hold. Then by conjugacy of $U$ and $V$, we get

$$
0=v(y, r)-u(x, q)+x y+\int_{0}^{T} q_{s} r_{s} d K_{s}
$$


Lemma 3.24 and [ET76, Proposition I.5.1] imply that $(y, r) \in \partial u(x, q) \cap \mathcal{E}$. Conversely, let $(x, q) \in \mathcal{K}$ and $(y, r) \in \mathcal{L} \cap \partial u(x, q)$. Then by [ET76, Proposition I.5.1] and Lemma 3.24, we deduce that (80) holds. Lemma 3.15 implies the finiteness of $u(x, q)$, which together with (80) results in the finiteness of $v(y, r)$, thus (75) holds and $(y, r) \in \mathcal{E}$. By Lemma 3.18, there exists a unique optimizer $\widehat{c}(x, q)$, for (2), and $\widehat{Y}(y, r)$, for (8), respectively. Therefore, from conjugacy of $U$ and $V$, Proposition 3.4, and (80), we obtain

$$
\begin{aligned}
& 0 \leq \mathbb{E}\left[\int_{0}^{T}\right.\left.\left(V\left(t, \widehat{Y}_{t}(y, r)\right)-U\left(t, \widehat{c}_{t}(x, q)\right)+\widehat{Y}_{t}(y, r) \widehat{c}_{t}(x, q)\right) d \kappa_{t}\right] \\
& \leq v(y, r)-u(x, q)+x y+\int_{0}^{T} q_{s} r_{s} d K_{s}=0
\end{aligned}
$$

This implies (76) and (177). This completes the proof of the theorem.

\section{Structure of the Dual Feasible Set}

By Assumption 4.1, there exists at most countable subset $\left(s_{k}\right)_{k \in \mathbb{N}}$ of $[0, T]$, where $\kappa$ has jumps. We define $\mathcal{D}^{\prime}$ the set of non-increasing, left-continuous and adapted processes $D$ that start at 1 and with the property that $D_{\theta_{0}}=1, D_{T} \underset{2^{n}}{\geq 0}$ and that, there exists some $n \in \mathbb{N}$ such that $D$ is constant off the discrete grid $\mathcal{T}_{n}:=\bigcup_{j=1}^{2^{n}}\left\{s_{j}\right\} \cup\left\{\frac{k}{2^{n}} T, k=0, \ldots, 2^{n}\right\}$.

Lemma 5.1. Let $\mathcal{G}^{\prime}:=\left\{Z D=\left(Z_{t} D_{t}\right)_{t \in[0, T]}: Z \in \mathcal{Z}^{\prime}, D \in \mathcal{D}^{\prime}\right\}$. Assume the conditions of Proposition 3.4 hold. Then $\mathcal{G}^{\prime}$ is convex.

Proof. Let $Z^{1} D^{1}$ and $Z^{2} D^{2}$ are the elements of $\mathcal{G}^{\prime}$ and let $\lambda \in(0,1)$ We need to show that $\lambda Z^{1} D^{1}+(1-\lambda) Z^{2} D^{2}=Z D$ for some $Z \in \mathcal{Z}^{\prime}$ and $D \in \mathcal{D}^{\prime}$. There exists $n \in \mathbb{N}$, such that $D^{1}$ and $D^{2}$ decrease at most on $\mathcal{T}_{n}$. Let $t_{k}$ 's be the elements of $\mathcal{T}_{n}$ arranged in an increasing order. Let us define $Z_{0}=D_{0}=1$ and for every $k \in\left\{0, \ldots, 2^{n}-1\right\}$, with

$$
A_{t}:=\lambda Z_{t_{k}}^{1} D_{t}^{1}+(1-\lambda) Z_{t_{k}}^{2} D_{t}^{2} \quad \text { and } \quad \alpha_{t}:=\frac{\lambda Z_{t_{k}}^{1} D_{t}^{1}}{A_{t}} 1_{\left\{A_{t} \neq 0\right\}}+\frac{\lambda Z_{t_{k}}^{1}}{\lambda Z_{t_{k}}^{1}+(1-\lambda) Z_{t_{k}}^{2}} 1_{\left\{A_{t}=0\right\}} \text {, }
$$

(note that $A_{t}=0$ if and only if both $D_{t}^{1}=0$ and $D_{t}^{2}=0$ ) we set

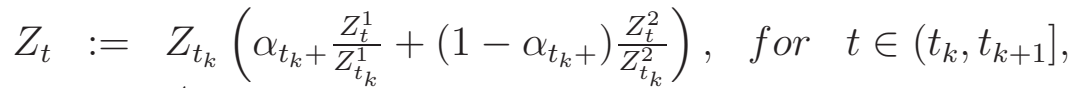

$$
\begin{aligned}
& D_{t}:=\frac{A_{t_{k}}}{Z_{t_{k}}}, \quad \text { for } t \in\left(t_{k}, t_{k+1}\right] \text {. }
\end{aligned}
$$

One can see that $Z D=\lambda Z^{1} D^{1}+(1-\lambda) Z^{2} D^{2}$ and that $Z \in \mathcal{Z}^{\prime}$, see e.g., [FK97, [Rok10], [Kar13], and [CT15] for discussions of the sets of processes with similar convexity-type 
properties to the one given in (81). To show that $D \in \mathcal{D}^{\prime}$, for $k \geq 1$, we observe that

$$
\begin{aligned}
& D_{t_{k}+}=\frac{A_{t_{k}+}}{Z_{t_{k}}} \\
& =\frac{\lambda Z_{t_{k}}^{1} D_{t_{k}+}^{1}+(1-\lambda) Z_{t_{k}}^{2} D_{t_{k}+}^{2}}{Z_{t_{k-1}}\left(\alpha_{t_{k-1}}+\frac{Z_{t_{k}}^{1}}{Z_{t_{k-1}}^{1}}+\left(1-\alpha_{t_{k-1}+}\right) \frac{Z_{t_{k}}^{2}}{Z_{t_{k-1}}^{2}}\right)} \\
& \leq \frac{\lambda Z_{t_{k}}^{1} D_{t_{k}}^{1}+(1-\lambda) Z_{t_{k}}^{2} D_{t_{k}}^{2}}{Z_{t_{k-1}}\left(\alpha_{t_{k-1}}+\frac{Z_{t_{k}}^{1}}{Z_{t_{k-1}}^{1}}+\left(1-\alpha_{t_{k-1}+}\right) \frac{Z_{t_{k}}^{2}}{Z_{t_{k-1}}^{2}}\right)} \\
& =\frac{\lambda Z_{t_{k}}^{1} D_{t_{k}}^{1}+(1-\lambda) Z_{t_{k}}^{2} D_{t_{k}}^{2}}{Z_{t_{k-1}}\left(\frac{\lambda Z_{t_{k-1}}^{1} D_{t_{k}}^{1}}{A_{t_{k-1}+}} \frac{Z_{t_{k}}^{1}}{Z_{t_{k-1}}^{1}}+\frac{(1-\lambda) Z_{t_{k-1}}^{2} D_{t_{k}}^{2}}{A_{t_{k-1}}} \frac{Z_{t_{k}}^{2}}{Z_{t_{k-1}}^{2}}\right)} 1_{\left\{A_{t_{k-1}+>}>0\right\}}+0 \cdot 1_{\left\{A_{t_{k-1}+}=0\right\}}
\end{aligned}
$$

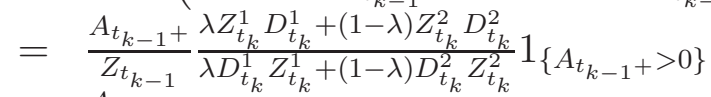

$$
\begin{aligned}
& =\frac{A_{t_{k-1}+}}{Z_{t_{k-1}}} 1_{\left\{A_{t_{k-1}+}>0\right\}} \\
& =D_{t_{k}} \text {. }
\end{aligned}
$$

Above, the inequality follows from the monotonicity of $D^{1}$ and $D^{2}$. Therefore, $D$ is nonincreasing. Also, clearly $D$ is nonnegative. Thus $Z D \in \mathcal{G}^{\prime}$.

The following lemma is an extension of Lemma 3.10 and amounts to a first layer of convexification of the set $\Upsilon$, i.e., of the budget constraints.

Lemma 5.2. Let the conditions of Proposition 3.4 hold, $(x, q) \in \mathcal{K}$, and $c$ is a nonnegative optional process. Then $c \in \mathcal{A}(x, q)$ if and only if

(82) $\mathbb{E}\left[\int_{0}^{T} c_{s} D_{s} Z_{s} d \kappa_{s}\right] \leq x+\mathbb{E}\left[\int_{0}^{T} q_{s} e_{s} D_{s} Z_{s} d \kappa_{s}\right], \quad$ for every $Z \in \mathcal{Z}^{\prime}$ and $D \in \mathcal{D}^{\prime}$.

Proof. The idea is to use the assertion of Lemma 3.10 and to approximate a given $D \in \mathcal{D}^{\prime}$ by (finite) linear combinations of the elements of $\Upsilon$, where $\Upsilon$ is defined in (15).

For a stopping time $\tau$, let us denote

$$
\Lambda^{\tau}:=1_{[0, \tau]} \in \Upsilon
$$

and fix $D \in \mathcal{D}^{\prime}$. Then there exists $l \in \mathbb{N}$, such that $D$ has has jumps at most on $\left\{t_{0}, t_{1}, \ldots, t_{l}\right\}$ for some increasing $t_{i}$ 's. For every $j \in\{0, \ldots, l\}, k \in\left\{0, \ldots 2^{n}\right\}$, and $n \in \mathbb{N}$, let us set

$$
\begin{aligned}
A_{k, n, j} & :=\left\{\omega: D_{t_{j}}(\omega)>0 \text { and } \frac{D_{t_{j}}(\omega)}{D_{t_{j}}(\omega)} \in\left(\frac{k-1}{2^{n}}, \frac{k}{2^{n}}\right]\right\}, \\
\tau^{k, n, j} & :=T 1_{A_{k, n, j}}+t_{j} 1_{A_{k, n, j}^{c}},
\end{aligned}
$$

Note that $D_{0}=1$ by definition of $\mathcal{D}^{\prime}, A_{k, n, j} \in \mathcal{F}_{t_{j}}$, and

$$
\frac{k-1}{2^{n}} \Lambda_{t_{j}+}^{\tau^{k, n, j}}=\left\{\begin{array}{lll}
\frac{k-1}{2^{n}} & \text { on } & A_{k, n, j} \\
0 & \text { on } & A_{k, n, j}^{c}
\end{array} .\right.
$$


By construction, we have

$$
D_{t_{j}+}=D_{t_{j}} \lim _{n \rightarrow \infty} \sum_{k=1}^{2^{n}} \frac{k-1}{2^{n}} \Lambda_{t_{j}+}^{\tau^{k, n, j}}
$$

where the sequence

$$
K_{t_{j}+}^{n}:=\sum_{k=1}^{2^{n}} \frac{k-1}{2^{n}} \Lambda_{t_{j}+}^{k, n, j}, \quad n \in \mathbb{N},
$$

is increasing on $\left\{D_{t_{j}}>0\right\}$, i.e.,

$$
K_{t_{j}+}^{n} 1_{\left\{D_{t_{j}}>0\right\}} \uparrow \frac{D_{t_{j}+}}{D_{t_{j}}} 1_{\left\{D_{t_{j}}>0\right\}} .
$$

Thus, for an arbitrary $j \in\{0, \ldots, l\}$, we have constructed a sequence of elements of $\Upsilon$, whose finite linear combinations monotonically increase to $\frac{D_{t_{j}}+}{D_{t_{j}}}$ on $\left\{D_{t_{j}}>0\right\}$ (i.e., if $j<l$, we have approximated $D$ on the interval $\left.\left(t_{j}, t_{j+1}\right]\right)$.

In order to construct a sequence that approximates $D$ at every point of its potential jumps, we first observe that for two stopping times $\tau$ and $\sigma$, we have

$$
\Lambda^{\tau} \Lambda^{\sigma}=1_{[0, \tau]} 1_{[0, \sigma]}=1_{[0, \tau \wedge \sigma]}=\Lambda^{\tau \wedge \sigma}
$$

Therefore, for every $n \in \mathbb{N}$,

$$
K_{t_{j}+}^{n} K_{t_{j+1}+}^{n}=\left(\sum_{k=1}^{2^{n}} \frac{k-1}{2^{n}} \Lambda_{t_{j}+}^{k, n, j}\right)\left(\sum_{k=1}^{2^{n}} \frac{k-1}{2^{n}} \Lambda_{t_{j+1}+}^{k, n, j+1}\right)=\sum_{i=1}^{4^{n}} \lambda^{n, i} \Lambda_{t_{j+1}+}^{\sigma_{n, i}},
$$

for some finite sequences of stopping times $\left(\sigma_{n, i}\right)_{i=1}^{4^{n}}$ and $[0,1)$-valued constants $\left(\lambda^{n, i}\right)_{i=1}^{4^{n}}$. Here $\Lambda^{\sigma_{n, i}}$ are such that for both $t=t_{j}$ and $t=t_{j+1}$ on $\left\{D_{t_{j+1}}>0\right\}$, we have

$$
\lim _{n \rightarrow \infty} \sum_{i=1}^{4^{n}} \lambda^{n, i} \Lambda_{t+}^{\sigma_{n, i}}=\frac{D_{t+}}{D_{t}}
$$

Similarly, with $r(t):=\max \left\{i: t_{i}<t\right\}$, let us define

$$
D_{t}^{n}:=\prod_{j=0}^{r(t)} K_{t_{j}+}^{n} 1_{\left\{D_{t_{j}}>0\right\}}, \quad t \in[0, T], n \in \mathbb{N} .
$$

As in (83), for every $n \in \mathbb{N}, D^{n}$ can be written as a finite linear combination of $\Lambda$ 's, such that $D_{t+}^{n} \uparrow D_{t+}$ for every $t \in\left\{t_{0}, \ldots, t_{l}\right\}$.

Finally, (82) can be obtained from Lemma 3.10 by the approximation of $D$ by $D^{n}$ 's as above and via the monotone convergence theorem (applied separately to $\left(e_{t}\right)^{+}$and $\left.\left(e_{t}\right)^{-}\right)$.

Corollary 5.3. Let the conditions of Proposition 3.4 hold. Then, for every pair $Z \in \mathcal{Z}^{\prime}$ and $D \in \mathcal{D}^{\prime}$, there exists $r^{Z D} \in \mathbb{L}^{1}(d K)$, such that $Z D \in \mathcal{Y}\left(1, r^{Z D}\right)$, where $\left(1, r^{Z D}\right) \in \mathcal{L}$. 
Proof. The existence of $r^{Z D}$, such that $Z D \in \mathcal{Y}\left(1, r^{Z D}\right)$ follows from Lemma 5.2 (equation (82)) and the approximation procedure in Lemma 5.2 (again, applied separately to $\left(e_{t}\right)^{+}$ and $\left.\left(e_{t}\right)^{-}\right)$combined with the monotone convergence theorem. As, the left-hand side in (82) is nonnegative, $\left(1, r^{Z D}\right) \in \mathcal{L}$.

For a given $Z \in \mathcal{Z}^{\prime}$ and $D \in \mathcal{D}^{\prime}$, let us recall that $r^{Z D}$ is given in Corollary 5.3 . We set

$$
\begin{gathered}
\mathcal{B}^{\prime}(x, q):=\left\{\left(y, y r^{Z D}\right) \in \mathcal{B}(x, q): y>0, Z \in \mathcal{Z}^{\prime}, D \in \mathcal{D}^{\prime}\right\}, \\
\mathcal{G}(x, q):=\left\{y Z^{\prime} D^{\prime} \in \mathcal{D}(x, q):\left(y, y r^{Z D}\right) \in \mathcal{B}^{\prime}(x, q)\right\}, \quad(x, q) \in \mathcal{K},
\end{gathered}
$$

Lemma 5.4. Let the conditions of Proposition 3.4 hold, then for every $(x, q) \in \mathcal{K}$, the closure of the convex, solid hull of $\mathcal{G}(x, q)$ in $\mathbb{L}^{0}$ coincides with $\mathrm{cl} \mathcal{D}(x, q)$.

Proof. Let $(x, q) \in \mathcal{K}$ be fixed. Along the lines of the proof of Lemma 3.20, one can show that

$$
(\mathcal{G}(x, q))^{o}=\mathcal{A}(x, q)
$$

Therefore,

$$
(\mathcal{G}(x, q))^{o o}=(\mathcal{A}(x, q))^{o}=\operatorname{cl} \mathcal{D}(x, q),
$$

where in the last equality we have used the conclusion of Lemma 3.20, As $\mathcal{G}(x, q) \subset$ $\operatorname{cl} \mathcal{D}(x, q)$, the assertion of the lemma follows from the bipolar theorem of Brannath and Schchermayer, [BS99, Theorem 1.3].

Corollary 5.5. Let the conditions of Proposition 3.4 hold and $(x, q) \in \mathcal{K}$. Then for every

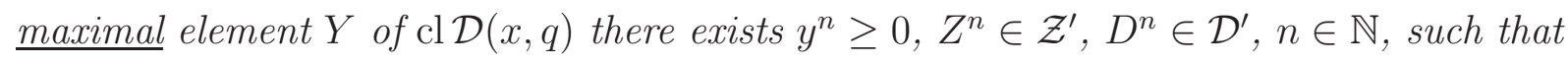
$\left(y^{n} Z^{n} D^{n}\right)_{n \in \mathbb{N}} \subset \mathcal{G}(x, q)$ and

$$
Y=\lim _{n \rightarrow \infty} y^{n} Z^{n} D^{n}, \quad(d \kappa \times \mathbb{P})-\text { a.e. and on } \bigcup_{n \in \mathbb{N}} \mathcal{T}_{n} .
$$

Proof. The $(d \kappa \times \mathbb{P})$-a.e. convergence follows from Lemma 5.4, By passing to subsequences of convex combinations, we also deduce the convergence on $\bigcup_{n \in \mathbb{N}} \mathcal{T}_{n}$.

Remark 5.6. It follows from Corollary 5.5 and Fatou's lemma that the maximal elements of $\operatorname{cl} \mathcal{D}(x, q)$ are strong supermartingales. Moreover, every maximal element of $\operatorname{cl} \mathcal{D}(x, q)$ is an optional strong supermartingale deflator, which is optional strong supermartingale $Y$, such that $X Y$ is an optional strong supermartingale for every $X \in \mathcal{X}(1)$. We refer to [DM82, Appendix 1] for a general characterization and to [CS16] for results on strong optional supermartingales as limits of martingales. The following section gives a more refined characterization of the dual minimizer. 


\section{Complementary Slackness}

For better readability of this section, we recall some notations and results that will be used below. Throughout this section, $(x, q) \in \mathcal{K}$ will be fixed, $\widehat{c}=\widehat{c}(x, q)$ is the optimizer to (2), $\widehat{V}$ is the corresponding wealth process, i.e.,

$$
\widehat{V}=x+\int_{0}^{\cdot} \widehat{H}_{s} d S_{s}-\int_{0}^{\cdot} \widehat{c}_{s} d \kappa_{s}+\int_{0}^{\cdot} q_{s} e_{s} d \kappa_{s}
$$

where $\widehat{H}$ is some $S$-integrable process, $\widehat{Y}$ be such that $\widehat{Y}_{t}=U^{\prime}(t, \widehat{c} t),(d \kappa \times \mathbb{P})$-a.e., i.e., $\widehat{Y}$ is the optimizer to (8) for some $(y, r) \in \mathcal{E} \cap \partial u(x, q), \widehat{Y} \in \mathcal{Y}(y, r)$ and

$$
\mathbb{E}\left[\int_{0}^{T} V\left(t, \widehat{Y}_{t}\right) d \kappa_{t}\right]=\inf _{Y \in \mathcal{Y}(y, r)} \mathbb{E}\left[\int_{0}^{T} V\left(t, Y_{t}\right) d \kappa_{t}\right] .
$$

By Corollary [5.5, $\widehat{Y}$ can be approximated by a sequence $y^{n} D^{n} Z^{n}, n \in \mathbb{N}$, where $y^{n}$ is a nonnegative constant, $D^{n} \in \mathcal{D}^{\prime}$, and $Z^{n} \in \mathcal{Z}^{\prime}, n \in \mathbb{N}$. In what follows, for any rightcontinuous increasing process $A$ satisfying

$$
A_{t}=0, \quad 0-\leq t \leq \theta_{0}-, \quad A_{T}=1
$$

i.e., for any probability measure $d A$ on the closed interval $\left[\theta_{0}, T\right]$ (extended to $[0, T]$ ) we will associate a process $D$ which is left-continuous and decreasing

$$
D_{t}:=1-A_{t-}=d A([t, T]), \quad 0 \leq t \leq T .
$$

One can also think that $D_{T+}=0$, although this is not necessary. It is clear that such $A \leftrightarrow D$ are in bijective correspondence. Below, all processes $A$ 's and $D$ 's (with indexes) will be in such bijective correspondence, except for the case of the limiting process $\widehat{A}$ (which is right-continuous) and the limiting process $\widehat{D}$ (that may be not left-continuous). The will be in a similar but more subtle correspondence. More precisely:

Theorem 6.1. Let the conditions of Theorem 4.3 hold. Let $\widehat{V}$ be the optimal wealth process, $\widehat{c}$ the optimal consumption, and $\widehat{Y}$ be the dual minimizer satisfying the assertions of Theorem 4.3. Then, there exists a strong supermartingale $\hat{Z}=\lim _{n \rightarrow \infty} Z^{n}$ (for $Z_{n} \in \mathcal{D}^{\prime}$, where the limit is in the sense of [CS16]) and a right-continuous increasing process $\widehat{A}$ with

$$
\widehat{A}_{t}=0 \quad \forall 0-\leq t \leq \theta_{0}-, \quad \widehat{A}_{T}=1,
$$

and a decreasing process $\widehat{D}$ with $\widehat{D}_{t}=1, \quad 0 \leq t \leq \theta_{0}$ and satisfying the complementary slackness condition

(85) $\left.\mathbb{P}\left(\widehat{D}_{t} \in\left[1-\widehat{A}_{t}, 1-\widehat{A}_{t-}\right], \forall \theta_{0} \leq t \leq T\right)\right)=1, \quad \mathbb{P}\left(\int_{\left[\theta_{0}, T\right)} 1_{\left\{\widehat{V}_{t-} \neq 0, \widehat{V}_{t} \neq 0\right\}} d \widehat{A}_{t}\right)=0$,

such that the dual minimizer can be decomposed as

$$
\hat{Y}=y \hat{Z} \hat{D}
$$


The proof of the Theorem 6.1 is split in several results.

Lemma 6.2. Let the conditions of Theorem 4.3 hold. With

$$
z:=x y+\int_{0}^{T} q_{s} r_{s} d K_{s}
$$

there exist $y^{n}>0, Z^{n} \in \mathcal{Z}^{\prime}$, and $D^{n} \in \mathcal{D}^{\prime}$, such that $y^{n} Z^{n} D^{n} \in z \mathcal{G}(x, q), n \in \mathbb{N}$, and

$$
\begin{gathered}
\widehat{Y}=\lim _{n \rightarrow \infty} y^{n} Z^{n} D^{n}, \quad(d \kappa \times \mathbb{P})-\text { a.e. } \\
y^{n} \rightarrow y>0
\end{gathered}
$$

For $A^{n}, n \in \mathbb{N}$, being in relation to $D^{n}$ exactly as described before Theorem 6.1 we have

$$
\mathbb{E}\left[\int_{\theta_{0}}^{T} \widehat{V}_{t} Z_{t}^{n} d A_{t}^{n}\right]=\mathbb{E}\left[Z_{T}^{n} \int_{\theta_{0}}^{T} \widehat{V}_{t} d A_{t}^{n}\right] \rightarrow 0 .
$$

Proof. Optimality of $\widehat{Y}$ and Corollary 5.5 imply (하 $)$. By (176) and Fatou's lemma, we get

$$
z=\mathbb{E}\left[\int_{0}^{T} \widehat{Y}_{s} \widehat{c}_{s} d \kappa_{s}\right] \leq \liminf _{n \rightarrow \infty} y^{n} \mathbb{E}\left[\int_{0}^{T} Z_{s}^{n} D_{s}^{n} \widehat{c}_{s} d \kappa_{s}\right]
$$

Let us fix $n \in \mathbb{N}$ and consider $\mathbb{E}\left[\int_{0}^{T} Z_{s}^{n} D_{s}^{n} \widehat{c}_{s} d \kappa_{s}\right]$. Using localization and integration by parts (along the lines of the proof of Lemma 3.7), we have

$$
\begin{aligned}
\mathbb{E}\left[\int_{0}^{T} Z_{s}^{n} D_{s}^{n} \widehat{c}_{s} d \kappa_{s}\right] & =\mathbb{E}\left[Z_{T}^{n} \int_{0}^{T} D_{s}^{n} \widehat{c}_{s} d \kappa_{s}\right] \\
& =\mathbb{E}\left[Z_{T}^{n} \int_{0}^{T}\left(1-A_{s-}^{n}\right) \widehat{c}_{s} d \kappa_{s}\right] \\
& =\mathbb{E}\left[Z_{T}^{n}\left(\int_{0}^{T} \widehat{c}_{s} d \kappa_{s}-\int_{0}^{T} A_{s-}^{n} \widehat{c}_{s} d \kappa_{s}\right)\right] \\
& =\mathbb{E}\left[Z_{T}^{n}\left(\int_{0}^{T} \widehat{c}_{s} d \kappa_{s}-A_{T}^{n} \int_{0}^{T} \widehat{c}_{s} d \kappa_{s}+\int_{0}^{T}\left(\int_{0}^{t} \widehat{c}_{s} d \kappa_{s}\right) d A_{t}^{n}\right)\right] \\
& =\mathbb{E}\left[Z_{T}^{n} \int_{0}^{T}\left(\int_{0}^{t} \widehat{c}_{s} d \kappa_{s}\right) d A_{t}^{n}\right] .
\end{aligned}
$$

The latter expression, using (84) and with $\bar{X}:=\|q\|_{\mathbb{L}^{\infty}(d K)} X^{\prime \prime}$ (where in turn $X^{\prime \prime}$ is given by the assertion $(v)$ of Lemma 3.2), we can rewrite as

$$
\mathbb{E}\left[Z_{T}^{n} \int_{0}^{T}\left(\left(x+\int_{0}^{t} \widehat{H}_{s} d S_{s}+\bar{X}_{t}\right)+\left(\int_{0}^{t} q_{s} e_{s} d \kappa_{s}-\bar{X}_{t}\right)-\widehat{V}_{t}\right) d A_{t}^{n}\right] .
$$

Let us denote

$$
\begin{aligned}
& T_{1}:=\mathbb{E}\left[Z_{T}^{n} \int_{0}^{T}\left(x+\int_{0}^{t} \widehat{H}_{s} d S_{s}+\bar{X}_{t}\right) d A_{t}^{n}\right], \\
& T_{2}:=\mathbb{E}\left[Z_{T}^{n} \int_{0}^{T}\left(\int_{0}^{t} q_{s} e_{s} d \kappa_{s}-\bar{X}_{t}\right) d A_{t}^{n}\right] .
\end{aligned}
$$

It follows from nonnegativity of $\widehat{V}$ in (84), Lemma 3.2, and nonnegativity of $\widehat{c}$ that

$$
\text { (90) } x+\int_{0}^{t} \widehat{H}_{s} d S_{s}+\bar{X}_{t} \geq \int_{0}^{t} \widehat{c}_{s} d \kappa_{s}+\bar{X}_{t}-\int_{0}^{t} q_{s} e_{s} d \kappa_{s} \geq \bar{X}_{t}-\int_{0}^{t} q_{s} e_{s} d \kappa_{s} \geq 0, \quad t \in[0, T],
$$

i.e., the integrand in $T_{1}$ is nonnegative. Let $\mathbb{Q}^{n}$ be the probability measure, whose density process with respect to $\mathbb{P}$ is $Z^{n}$. As $\left(x+\int_{0} \widehat{H}_{s} d S_{s}+\bar{X}\right)$ and $\bar{X}$ are local martingales under $\mathbb{Q}^{n}$, by [Pro04, Theorem III.27, p. 128], $A_{-}^{n} \cdot\left(x+\int_{0}^{\cdot} \widehat{H}_{s} d S_{s}+\bar{X}\right)$ and $A_{-}^{n} \cdot \bar{X}$ are local 
martingales. Let $\left(\tau_{k}\right)_{k \in \mathbb{N}}$ be a localizing sequence for both $A_{-}^{n} \cdot\left(x+\int_{0}^{\cdot} \widehat{H}_{s} d S_{s}+\bar{X}\right)$ and $A_{-}^{n} \cdot \bar{X}$. By the monotone convergence theorem and integration by parts, we get

$$
\begin{aligned}
T_{1} & =\lim _{k \rightarrow \infty} \mathbb{E}^{\mathbb{Q}^{n}}\left[\int_{0}^{\tau_{k}}\left(x+\int_{0}^{t} \widehat{H}_{s} d S_{s}+\bar{X}_{t}\right) d A_{t}^{n}\right] \\
& =\lim _{k \rightarrow \infty}\left(\mathbb{E}^{\mathbb{Q}^{n}}\left[\int_{0}^{\tau_{k}}\left(-A_{t-}^{n}\right) d\left(x+\int_{0}^{t} \widehat{H}_{s} d S_{s}+\bar{X}_{t}\right)+A_{\tau_{k}}^{n}\left(x+\int_{0}^{\tau_{k}} \widehat{H}_{s} d S_{s}+\bar{X}_{\tau_{k}}\right)\right]\right) \\
& =\lim _{k \rightarrow \infty} \mathbb{E}^{\mathbb{Q}^{n}}\left[A_{\tau_{k}}^{n}\left(x+\int_{0}^{\tau_{k}} \widehat{H}_{s} d S_{s}+\bar{X}_{\tau_{k}}\right)\right] .
\end{aligned}
$$

Let us consider $T_{2}$. With $\mathcal{E}^{q}:=\int_{0}^{\cdot} q_{s} e_{s} d \kappa_{s}$, Lemma 3.2 implies positivity of $\mathcal{E}_{t}^{q}-\bar{X}_{t}, t \in$ $[0, T]$, which in turn allows to invoke the monotone convergence theorem, and we obtain

$$
\begin{aligned}
T_{2} & =\mathbb{E}^{\mathbb{Q}^{n}}\left[\int_{0}^{T}\left(\mathcal{E}_{t}^{q}-\bar{X}_{t}\right) d A_{t}^{n}\right] \\
& =\lim _{k \rightarrow \infty} \mathbb{E}^{\mathbb{Q}^{n}}\left[\int_{0}^{\tau_{k}} \mathcal{E}_{t}^{q} d A_{t}^{n}-\int_{0}^{\tau_{k}} \bar{X}_{t} d A_{t}^{n}\right] .
\end{aligned}
$$

By positivity of $\bar{X}$ and the monotone convergence theorem, we have

$$
\lim _{k \rightarrow \infty} \mathbb{E}^{\mathbb{Q}^{n}}\left[\int_{0}^{\tau_{k}} \bar{X}_{t} d A_{t}^{n}\right]=\mathbb{E}^{\mathbb{Q}^{n}}\left[\int_{0}^{T} \bar{X}_{t} d A_{t}^{n}\right]
$$

Therefore, $\lim _{k \rightarrow \infty} \mathbb{E}^{\mathbb{Q}^{n}}\left[\int_{0}^{\tau_{k}} \mathcal{E}_{t}^{q} d A_{t}^{n}\right]=\mathbb{E}^{\mathbb{Q}^{n}}\left[\int_{0}^{T} \mathcal{E}_{t}^{q} d A_{t}^{n}\right]$. We deduce that

$$
\mathbb{E}^{\mathbb{Q}^{n}}\left[\int_{0}^{\tau_{k}} \bar{X}_{t} d A_{t}^{n}\right]=\mathbb{E}^{\mathbb{Q}^{n}}\left[-\left(A_{-}^{n} \cdot \bar{X}\right)_{\tau_{k}}+\bar{X}_{\tau_{k}} A_{\tau_{k}}^{n}\right]=\mathbb{E}^{\mathbb{Q}^{n}}\left[\bar{X}_{\tau_{k}} A_{\tau_{k}}^{n}\right] .
$$

Whereas, in the other term in $T_{2}$, we get

$$
\begin{aligned}
\mathbb{E}^{\mathbb{Q}^{n}}\left[\int_{0}^{T} \mathcal{E}_{t}^{q} d A_{t}^{n}\right] & =\mathbb{E}^{\mathbb{Q}^{n}}\left[\mathcal{E}_{T}^{q} A_{T}^{n}-\left(A_{-}^{n} \cdot \mathcal{E}^{q}\right)_{T}\right] \\
& =\mathbb{E}^{\mathbb{Q}^{n}}\left[\mathcal{E}_{T}^{q}-\left(A_{-}^{n} \cdot \mathcal{E}^{q}\right)_{T}\right] \\
& =\mathbb{E}^{\mathbb{Q}^{n}}\left[\left(\left(1-A_{-}^{n}\right) \cdot \mathcal{E}^{q}\right)_{T}\right] \\
& =\mathbb{E}^{\mathbb{Q}^{n}}\left[\left(D^{n} \cdot \mathcal{E}^{q}\right)_{T}\right] .
\end{aligned}
$$

using integration by parts and localization, as in the proof of Lemma 3.7, we can rewrite the latter expression as

$$
\mathbb{E}\left[\int_{0}^{T} D_{s}^{n} Z_{s}^{n} q_{s} e_{s} d \kappa_{s}\right]
$$

We conclude that

$$
T_{2}=-\lim _{k \rightarrow \infty} \mathbb{E}^{\mathbb{Q}^{n}}\left[\bar{X}_{\tau_{k}} A_{\tau_{k}}^{n}\right]+\mathbb{E}\left[\int_{0}^{T} D_{s}^{n} Z_{s}^{n} q_{s} e_{s} d \kappa_{s}\right] .
$$

Combining this with (91), we obtain

$$
T_{1}+T_{2}=\mathbb{E}\left[\int_{0}^{T} D_{s}^{n} Z_{s}^{n} q_{s} e_{s} d \kappa_{s}\right]+\lim _{k \rightarrow \infty} \mathbb{E}^{\mathbb{Q}^{n}}\left[A_{\tau_{k}}^{n}\left(x+\int_{0}^{\tau_{k}} \widehat{H}_{s} d S_{s}+\bar{X}_{\tau_{k}}\right)\right]-\lim _{k \rightarrow \infty} \mathbb{E}^{\mathbb{Q}^{n}}\left[A_{\tau_{k}}^{n} \bar{X}_{\tau_{k}}\right]
$$


As both limits in the right-hand side exist and by positivity of $\left(x+\int_{0}^{\tau_{k}} \widehat{H}_{s} d S_{s}+\bar{X}_{\tau_{k}}\right)$, established in (90), we can bound the difference of the limits as

$$
\begin{aligned}
& \lim _{k \rightarrow \infty} \mathbb{E}^{\mathbb{Q}^{n}}\left[A_{\tau_{k}}^{n}\left(x+\int_{0}^{\tau_{k}} \widehat{H}_{s} d S_{s}+\bar{X}_{\tau_{k}}\right)\right]-\lim _{k \rightarrow \infty} \mathbb{E}^{\mathbb{Q}^{n}}\left[A_{\tau_{k}}^{n} \bar{X}_{\tau_{k}}\right] \\
& \quad \leq \lim _{k \rightarrow \infty} \mathbb{E}^{\mathbb{Q}^{n}}\left[x+\int_{0}^{\tau_{k}} \widehat{H}_{s} d S_{s}+\bar{X}_{\tau_{k}}\right]-\lim _{k \rightarrow \infty} \mathbb{E}^{\mathbb{Q}^{n}}\left[A_{\tau_{k}}^{n} \bar{X}_{\tau_{k}}\right] \\
& \leq \lim _{k \rightarrow \infty} \mathbb{E}^{\mathbb{Q}^{n}}\left[x+\int_{0}^{\tau_{k}} \widehat{H}_{s} d S_{s}\right]+\lim _{k \rightarrow \infty} \mathbb{E}^{\mathbb{Q}^{n}}\left[\left(1-A_{\tau_{k}}^{n}\right) \bar{X}_{\tau_{k}}\right] .
\end{aligned}
$$

By definition of $\mathcal{M}^{\prime}, \bar{X}$ is a uniformly integrable martingale under $\mathbb{Q}^{n}$. Therefore, as $\left(1-A_{\tau_{k}}^{n}\right)$ is bounded, we can pass the limit inside of the expectation to obtain $\lim _{k \rightarrow \infty} \mathbb{E}^{\mathbb{Q}^{n}}\left[\left(1-A_{\tau_{k}}^{n}\right) \bar{X}_{\tau_{k}}\right]=0$. In turn $x+\int_{0} \widehat{H}_{s} d S_{s}$ is a supermartingale under $\mathbb{Q}^{n}$ (see the argument in the proof of Lemma 3.77). We conclude that

$$
T_{1}+T_{2} \leq x+\mathbb{E}\left[\int_{0}^{T} D_{s}^{n} Z_{s}^{n} q_{s} e_{s} d \kappa_{s}\right]=x+\int_{0}^{T} q_{s} \rho_{s}^{n} d K_{s},
$$

for some $\rho^{n}$, which is well-defined by Corollary [5.3, and such that $y^{n}\left(1, \rho^{n}\right) \in z \mathcal{B}(x, q)$, since $y^{n} Z^{n} D^{n} \in z \mathcal{G}(x, q)$. Thus, from (88) and (89), we get

$$
z \leq\left(x+\int_{0}^{T} q_{s} \rho_{s}^{n} d K_{s}\right) y^{n}-\lim _{n \rightarrow \infty} y^{n} \mathbb{E}\left[Z_{T}^{n} \int_{0}^{T} \widehat{V}_{t} d A_{t}^{n}\right] .
$$

By optimality of $\widehat{Y}, z \geq\left(x+\int_{0}^{T} q_{s} \rho_{s}^{n} d K_{s}\right) y^{n} \geq 0$. Therefore, by nonnegativity of $\mathbb{E}\left[Z_{T}^{n} \int_{0}^{T} \widehat{V}_{t} d A_{t}^{n}\right]$, and since $y^{n}$ converges to a strictly positive limit, we conclude that

$$
\lim _{n \rightarrow \infty} \mathbb{E}\left[Z_{T}^{n} \int_{0}^{T} \widehat{V}_{t} d A_{t}^{n}\right]=0 .
$$

Applying integration by parts and localization we deduce the assertion of the lemma.

Remark 6.3. We emphasize again that $d A^{n}$ are probability measures on $\left[\theta_{0}, T\right]$, which can have mass at the endpoints, and $D_{t}^{n}=1-A_{t-}^{n}, \theta_{0} \leq t \leq T, D_{T+}^{n}=0$.

In the subsequent part, we will follow the notations of Lemma 6.2 and we will work with a further subsequence, still denoted by $n$, such that $y_{n} \rightarrow y>0$,

$$
Z^{n} D^{n} \rightarrow \frac{\widehat{Y}}{y}, \quad(d \kappa \times \mathbb{P})-a . e,
$$

and

$$
\sum_{k=n}^{\infty} \mathbb{E}\left[Z_{T}^{n} \int_{0}^{T} \widehat{V}_{t} d A_{t}^{n}\right] \leq \frac{2^{-n}}{n}
$$

where the existence of a subsequence satisfying (93) follows from (87). The next results is a Komlos-type lemma, largely based on the results in [CS16], applied to the doublesequence of processes $\left(Z^{n},\left(D^{n}\right)^{-1}\right)$. We observe that the process $\left(D^{n}\right)^{-1}$ takes values in $[1, \infty]$ is increasing, left-continuous, and satisfies

$$
\left(D_{t}^{n}\right)^{-1}=1, \quad 0 \leq t \leq \theta_{0} .
$$


Lemma 6.4. Let the conditions of Theorem 4.3 hold. In the notations of Lemma 6.2, for each $n$, there exist a finite index $N(n)$ and convex weights

$$
\alpha_{n, k} \geq 0, \quad k=n, \ldots, N(n), \quad \sum_{k=n}^{N(n)} \alpha_{n, k}=1,
$$

and there exists a strong optional super-martingale $\widehat{Z}$ and a non-decreasing (not necessarily left-continuous) process $\widehat{D}$ with $\widehat{D}_{t}=0, \forall 0 \leq t \leq \theta_{0}$, such that, simultaneously,

(1) $\tilde{Z}^{n}:=\sum_{k=n}^{N(n)} \alpha_{n, k} Z^{k} \rightarrow \widehat{Z}$ in the sense of [CS16] i.e. for any stopping time $0 \leq \tau \leq$ $T$ we have

$$
\tilde{Z}_{\tau}^{n} \longrightarrow{ }^{\mathbb{P}} \widehat{Z}_{\tau}
$$

and

(2)

$$
\mathbb{P}\left(\sum_{k=n}^{N(n)} \alpha_{n, k}\left(D_{t}^{k}\right)^{-1} \rightarrow\left(\widehat{D}_{t}\right)^{-1}, \quad \forall 0 \leq t \leq T+\right)=1
$$

We have set $D_{T+}=D_{T+}^{k}=0$.

Furthermore, with the notation

$$
\widehat{A}_{t}=1-\widehat{D}_{t+}, \quad 0 \leq t \leq T, \quad \widehat{A}_{0-}=0,
$$

we have the probability measure $d \widehat{A}$ on $\left[\theta_{0}, T\right]$ such that

$$
\left.\mathbb{P}\left(\widehat{D}_{t} \in\left[1-\widehat{A}_{t}, 1-\widehat{A}_{t-}\right], \forall \theta_{0} \leq t \leq T\right)\right)=1 .
$$

Denoting by

$\tilde{A}_{t}^{n}:=1-\left(\sum_{k=n}^{N(n)} \alpha_{n, k}\left(1-A_{t}^{k}\right)^{-1}\right)^{-1}=1-\left(\sum_{k=n}^{N(n)} \alpha_{n, k}\left(D_{t+}^{k}\right)^{-1}\right)^{-1}, \quad 0 \leq t \leq T, \quad \tilde{A}_{0-}^{n}=0$, the point-wise convergence in time (at all times where there is continuity) additionally implies

$$
d \tilde{A}^{n} \rightarrow d \widehat{A}, \quad \mathbb{P}-\text { a.e. }
$$

in the sense of weak convergence of probability measures on $\left[\theta_{0}, T\right]$.

Proof. The proof reduces to applying the Komlos-type results in CS16] to the sequence $Z^{n}$ and [CS06, Proposition 3.4] to the sequence $\left(D^{n}\right)^{-1}$, simultaneously. We observe that:

(1) first, no bounds are needed for $D^{-1}$ 's since we can apply the unbounded Komlos lemma in [DS94, Lemma A1.1] (and Remark 1 following it) to the proof from [CS06, Propositions 3.4], and this works even for infinite values (according to Remark 1 after [DS94, Lemma A1.1]). Also, predictability can be replaced by optionality, without any change to the proof. 
(2) the Komlos arguments can be applied to both sequences $Z^{n}$ and $D^{n}$ simultaneously, with the same convex weights. In order to do this, we first apply Komlos to one sequence, then replace both original sequences by their convex combinations with the weights just obtained, and then apply Komlos again for the other sequence, and update the convex weight to both sequences.

Corollary 6.5. Let the conditions of Theorem 4.3 hold. In the notations of Lemma 6.4. we have the representation

$$
\widehat{Y}=y \widehat{Z} \widehat{D}, \quad(d \kappa \times \mathbb{P})-\text { a.e. }
$$

Proof. Consider an observation that, for non-negative numbers $a_{k}, b_{k}, k=n, \ldots, N(n)$, we have

$$
\min _{k=n, \ldots, N(n)}\left(\frac{a_{k}}{b_{k}}\right) \leq \frac{\sum_{k=n}^{N(n)} \alpha_{n, k} a^{k}}{\sum_{k=n}^{N(n)} \alpha_{n, k} b_{k}} \leq \max _{k=n, \ldots, N(n)}\left(\frac{a_{k}}{b_{k}}\right) .
$$

We apply this to $a_{k}=Z^{k}, b_{k}=\left(D^{k}\right)^{-1}$, to obtain

$$
\min _{k=n, \ldots, N(n)}\left(Z^{k} D^{k}\right) \leq \frac{\sum_{k=n}^{N(n)} \alpha_{n, k} Z^{k}}{\sum_{k=n}^{N(n)} \alpha_{n, k}\left(D^{k}\right)^{-1}}=\tilde{Z}^{n} \tilde{D}^{n} \leq \max _{k=n, \ldots, N(n)}\left(Z^{k} D^{k}\right),
$$

pointwise a.e. in the product space, where $\tilde{D}^{n}:=\frac{1}{\sum_{k=n}^{N(n)} \alpha_{n, k}\left(D^{k}\right)^{-1}}$. Since $Z^{n} D^{n} \rightarrow \widehat{Y} / y$, $(d \kappa \times \mathbb{P})$-a.e., we conclude that both $\left(\min _{k=n, \ldots, N(n)}\left(Z^{k} D^{k}\right)\right)_{n \in \mathbb{N}}$ and $\left(\max _{k=n, \ldots, N(n)}\left(Z^{k} D^{k}\right)\right)_{n \in \mathbb{N}}$ converge to $\frac{\widehat{Y}}{y},(d \kappa \times \mathbb{P})$-a.e., therefore

$$
\tilde{Z}^{n} \tilde{D}^{n} \rightarrow \frac{\widehat{Y}}{y}, \quad(d \kappa \times \mathbb{P})-\text { a.e. }
$$

Using (92) above, if we did not plan to identify the limit $\widehat{Z}$ as a strong-supermartingale, but only as a $(d \kappa \times \mathbb{P})$-a.e. limit in the product space, we could only apply Komlos arguments to the single sequence $D^{-1}$, to conclude convergence of the other convex combination $\tilde{Z}^{n}$ to $\frac{\widehat{Y}}{y \widehat{D}}$, defined up to a.e. equality in the product space.

With our (stronger) double Komlos argument, we have that, in addition to (92) we have

$$
\widehat{Z}_{\tau} \widehat{D}_{\tau}=\mathbb{P}-\lim _{n} \tilde{Z}_{\tau}^{n} \tilde{D}_{\tau}^{n}, \quad \text { for every }[0, T] \text {-valued stopping time } \tau,
$$

(where $\widehat{Z}$ is a strong supermartingale, and $\widehat{D}$ is well defined at all times).

It remains to prove that $\widehat{Y} / y=\widehat{Z} \widehat{D},(d \kappa \times \mathbb{P})$-a.e. We point out that the convergence (97) is topological. Let us consider an arbitrary optional set $O \subset \Omega \times[0, T]$ and fix an upper bound $M$. It follows from (96) that

$$
1_{O} \min \left\{\tilde{Z}^{n} \tilde{D}^{n}, M\right\} \rightarrow 1_{O} \min \left\{\frac{\widehat{Y}}{y}, M\right\}, \quad(d \kappa \times \mathbb{P})-\text { a.e. }
$$


Therefore, we get

$$
\mathbb{E}\left[\int_{0}^{T} 1_{O}(t, \cdot) \min \left\{\tilde{Z}_{t}^{n} \tilde{D}_{t}^{n}, M\right\} d \kappa_{t}\right] \rightarrow \mathbb{E}\left[\int_{0}^{T} 1_{O}(t, \cdot) \min \left\{\frac{\widehat{Y}_{t}}{y}, M\right\} d \kappa_{t}\right] .
$$

Recall that the stochastic clock has a density $d \kappa_{t}=\varphi_{t} d K_{t}$ with respect to the deterministic clock $d K_{t}$. For each fixed $t$, from (97) we have

$$
1_{O}(t, \cdot) \min \left\{\tilde{Z}_{t}^{n} \tilde{D}_{t}^{n}, M\right\} \varphi_{t} \rightarrow 1_{O}(t, \cdot) \min \left\{\widehat{Z}_{t} \widehat{D}_{t}, M\right\} \varphi_{t}, \quad \text { in }-\mathbb{P} .
$$

Recalling that $\mathbb{E}\left[\varphi_{t}\right]<\infty$ we have, for fixed $t$, that

$$
M \times \mathbb{E}\left[\varphi_{t}\right] \geq \mathbb{E}\left[1_{O}(t, \cdot) \min \left\{\tilde{Z}_{t}^{n} \tilde{D}_{t}^{n}, M\right\} \varphi_{t}\right] \rightarrow \mathbb{E}\left[1_{O}(t, \cdot) \min \left\{\widehat{Z}_{t} \widehat{D}_{t}, M\right\} \varphi_{t}\right]
$$

We integrate the above with respect to the deterministic clock $d K_{t}$ to obtain

$$
\begin{aligned}
& \mathbb{E}\left[\int_{0}^{T} 1_{O}(t, \cdot) \min \left\{\tilde{Z}_{t}^{n} \tilde{D}_{t}^{n}, M\right\} d \kappa_{t}\right]=\int_{0}^{T} \mathbb{E}\left[1_{O}(t, \cdot) \min \left\{\tilde{Z}_{t}^{n} \tilde{D}_{t}^{n}, M\right\} \varphi_{t}\right] d K_{t} \rightarrow \\
& \quad \rightarrow \int_{0}^{T} \mathbb{E}\left[1_{O}(t, \cdot) \min \left\{\widehat{Z}_{t} \widehat{D}_{t}, M\right\} \varphi_{t}\right] d K_{t}=\mathbb{E}\left[\int_{0}^{T} 1_{O}(t, \cdot) \min \left\{\widehat{Z}_{t} \widehat{D}_{t}, M\right\} d \kappa_{t}\right]
\end{aligned}
$$

Together with (98) we have

$$
\mathbb{E}\left[\int_{0}^{T} 1_{O}(t, \cdot) \min \left\{\frac{\widehat{Y}_{t}}{\hat{y}}, M\right\} d \kappa_{t}\right]=\mathbb{E}\left[\int_{0}^{T} 1_{O}(t, \cdot) \min \left\{\widehat{Z}_{t} \widehat{D}_{t}, M\right\} d \kappa_{t}\right],
$$

which holds for any optional set $O$ and any bound $M$, therefore $\widehat{Y}=y \widehat{Z} \widehat{D},(d \kappa \times \mathbb{P})$ a.e.

Proof of the Theorem 6.1. Since

$$
\sum_{k=n}^{N(n)} \alpha_{n, k}\left(D_{t}^{k}\right)^{-1} \rightarrow\left(\widehat{D}_{t}\right)^{-1}, \quad t \in[0, T]
$$

recalling that $\widehat{A}$ was defined from $\widehat{D}$ and (95). As the processes $A^{n}$, therefore $\tilde{A}^{n}$ only increase by jumps. Therefore we get

$$
\begin{aligned}
& \Delta \tilde{A}_{s}^{n}=\frac{1}{\sum_{k=n}^{N(n)} \alpha_{n, k} \frac{1}{D_{s}^{k}}}-\frac{1}{\sum_{k=n}^{N(n)} \alpha_{n, k} \frac{1}{D_{s+}^{k}}} \\
& =\sum_{k=n}^{N(n)} \frac{\alpha_{n, k} \frac{1}{D_{s+}^{k}}}{\sum_{\leq 1}^{N(n)} \alpha_{n, k} \frac{1}{D_{s+}^{k}}} \frac{\Delta A_{s}^{k}}{D_{s}^{k}} \underbrace{\frac{1}{\sum_{k=n}^{N(n)} \alpha_{n, k} \frac{1}{D_{s}^{k}}}}_{\leq 1} .
\end{aligned}
$$


As $\frac{\alpha_{n, k} \frac{1}{D_{s+}^{k}}}{\left(\sum_{k=n}^{N(n)} \alpha_{n, k} \frac{1}{D_{s+}^{k}}\right)} \leq 1$ and $\sum_{k=n}^{N(n)} \alpha_{n, k} \frac{1}{D_{s}^{k}} \geq 1$, we can bound the latter term in (100) by $\sum_{k=n}^{N(n)} \frac{\Delta A_{s}^{k}}{D_{s}^{k}}$ for $s \in[0, T]$. We deduce that

$$
\Delta \tilde{A}_{s}^{n} \leq \sum_{k=n}^{N(n)} \frac{\Delta A_{s}^{k}}{D_{s}^{k}}, \quad s \in[0, T]
$$

Therefore, we have

$$
\int_{0}^{t} \widehat{V}_{u} d \tilde{A}_{u}^{n} \leq \sum_{k=n}^{N(n)}\left(D_{t}^{k}\right)^{-1} \int_{0}^{t} \widehat{V}_{u} d A_{u}^{k}, \quad t \in[0, T]
$$

and thus, we obtain

$$
\left(\min _{k=n, \ldots, N(n)}\left(Z_{t}^{k} D_{t}^{k}\right)\right) \int_{0}^{t} \widehat{V}_{u} d \tilde{A}_{u}^{n} \leq \sum_{k=n}^{N(n)}\left(Z_{t}^{k} D_{t}^{k}\right)\left(D_{t}^{k}\right)^{-1} \int_{0}^{t} \widehat{V}_{u} d A_{u}^{k}=\sum_{k=n}^{N(n)} Z_{t}^{k} \int_{0}^{t} \widehat{V}_{u} d A_{u}^{k}, \quad t \in[0, T] .
$$

Since the process

$$
L_{t}^{n}:=\sum_{k=n}^{N(n)} Z_{t}^{k} \int_{0}^{t} \widehat{V}_{u} d A_{u}^{k}, \quad 0 \leq t \leq T
$$

is a non-negative right-continuous submartingale, the maximal inequality and (93) together imply

so

$$
\mathbb{P}\left(\sup _{0 \leq t \leq T} L_{t}^{n} \geq \frac{1}{n}\right) \leq n \mathbb{E}\left[L_{T}^{n}\right] \leq 2^{-n}
$$

$$
\sup _{0 \leq t \leq T} L_{t}^{n} \rightarrow 0, \quad \mathbb{P}-\text { a.s. }
$$

Since $Z^{n} D^{n} \rightarrow \frac{1}{y} \widehat{Y}>0,(d \kappa \times \mathbb{P})$-a.e., consequenlty

$$
\min _{k=n, \ldots, N(n)}\left(Z^{k} D^{k}\right) \rightarrow \frac{1}{y} \widehat{Y}>0 \quad(d \kappa \times \mathbb{P})-\text { a.e. }
$$

we obtain from(101) and (102) that the increasing RC process

$$
\tilde{L}_{t}^{n}:=\int_{0}^{t} \widehat{V}_{u} d \tilde{A}_{u}^{n}, \quad 0 \leq t \leq T,
$$

converges to zero in the product space. Denoting by $\mathcal{O} \subset \Omega \times[0, T]$ the exceptional set where convergence to zero does not take place, and taking into account that $\tilde{L}^{n}$ are increasing, we have that for for

$$
T^{\mathcal{O}}(\omega)=\inf \{0 \leq t \leq T:(\omega, t) \in \mathcal{O}\}
$$

we have

$$
\left(T^{\mathcal{O}}, T\right] \subset \mathcal{O}
$$


Now

$$
(d \kappa \times \mathbb{P})(\mathcal{O})=0,
$$

implies that $T^{\mathcal{O}} \geq T, \mathbb{P}$-a.s. (here used an assumption that $T$ is the minimal time horizon in the sense that the deterministic clock $K$ is such that $K_{t}<K_{T}$, for every $t \in[0, T)$, this assumption does not restrict generality). Thus, there exists a a set $\Omega^{*}$ of full probability $\mathbb{P}\left(\Omega^{*}\right)=1$ such that, for each $\omega \in \Omega^{*}$ and $t<T$ we have

$$
\int_{0}^{t} \widehat{V}_{u}(\omega) d \tilde{A}_{t}^{n}(\omega) \rightarrow 0
$$

Let us fix an $\omega \in \Omega^{*}$ and such that, for this $\omega$, the probability measure $d \tilde{A}^{n}(\omega)$ converges weakly to $d \widehat{A}(\omega)$ over the interval $\left[\theta_{0}(\omega), T\right]$. The set of such $\omega$ 's still has probability 1 . The Skorokhod representation theorem asserts that there exists a new probability space $\Omega^{\omega}$ and a sequence of random times $\left(t^{n}(\omega)\right)_{n \in \mathbb{N}}$ as well as $\widehat{t}(\omega)$ such that the distribution of $t^{n}(\omega)$ is $d \tilde{A}^{n}$, the distribution of $\widehat{t}(\omega)$ is $d \widehat{A}(\omega)$ and

$$
t^{n}(\omega) \rightarrow \widehat{t}(\omega), \quad \mathbb{P}^{\omega}-\text { a.s. }
$$

on the new, artificial, probability space. Fix $t<T$. We have

$$
\mathbb{E}^{\omega}\left[\widehat{V}_{t^{n}(\omega)}(\omega) 1_{\left\{t^{n}(\omega) \leq t\right\}}\right]=\int_{\theta_{0}}^{t} \widehat{V}_{u}(\omega) d \tilde{A}_{u}^{n}(\omega) \rightarrow 0 .
$$

One can see that

$$
\xi(\omega):=\liminf _{n} \widehat{V}_{t^{n}(\omega)}(\omega) \in\left\{\widehat{V}_{\widehat{t}(\omega)-}(\omega), \widehat{V}_{\widehat{t}(\omega)}(\omega)\right\}, \quad \mathbb{P}^{\omega}-a . s .
$$

and

$$
1_{\left\{t^{n}(\omega) \leq t\right\}} \rightarrow 1_{\{\widehat{t}(\omega) \leq t\}} \text { on }\{\widehat{t}(\omega)<t\}, \mathbb{P}^{\omega}-\text { a.s. }
$$

Therefore, applying Fatou's lemma on $\Omega^{\omega}$, we obtain

$$
\mathbb{E}^{\omega}\left[\xi(\omega) 1_{\{\widehat{t}(\omega)<t\}}\right]=0 .
$$

We recall that both $\widehat{V}(\omega)$ and $\widehat{V}_{-}(\omega)$ are nonnegative, consequently we have

$$
0 \leq \min \left\{\widehat{V}_{\widehat{t}(\omega)-}(\omega), \widehat{V}_{\widehat{t}(\omega)}(\omega)\right\} \leq \xi(\omega),
$$

and therefore we get

$$
\mathbb{E}^{\omega}\left[\min \left\{\widehat{V}_{\widehat{t}(\omega)-}(\omega), \widehat{V}_{\widehat{t}(\omega)}(\omega)\right\} 1_{\{\widehat{t}(\omega)<t\}}\right]=0 .
$$

This means that the distribution of $\widehat{t}(\omega)$ over the interval $\left[\theta_{0}(\omega), t\right)$ only charges the complement of the set of times

$$
\left\{u<t: \quad \widehat{V}_{u-}(\omega)=0 \quad \text { or } \quad \widehat{V}_{u}(\omega)=0\right\} .
$$

By taking $t \rightarrow T$, one completes the proof. 


\section{REFERENCES}

[BK17] P. Bank and H. Kauppila. Convex duality for stochastic singular control problems. Ann. Appl. Probab., 27(1):485-516, 2017.

[BP12] V. Barbu and T. Precupanu. Convexity and Optimization in Banach Spaces. Springer, 4th edition, 2012.

[BS99] W. Brannath and W. Schachermayer. A bipolar theorem for subsets of $L_{+}^{0}(\Omega, \mathcal{F}, \mathbb{P})$. Séminaire de Probabilités XXXIII, Springer Lecture Notes in Mathematics, XXXIII:349-354, 1999.

[CS06] L. Campi and W. Schachermayer. A super-replication theorem in Kabanov's model of transaction costs. Finance Stoch., 10(4):579-596, 2006.

[CS16] C. Czichowsky and W. Schachermayer. Strong supermartingales and limits of non-negative martingales. Ann. Probab., 44(1):171-205, 2016.

[CSW01] J. Cvitanić, W. Schachermayer, and H. Wang. Utility maximization in incomplete markets with random endowment. Finance Stoch., 5:259-272, 2001.

[CT15] C. Cuchiero and J. Teichmann. A convergence result for the Emery topology and a variant of the proof of the fundamental theorem of asset pricing. Finance Stoch., 19(4):743-761, 2015.

[Cuo97] D. Cuoco. Optimal consumption and equilibrium prices with portfolio constraints and stochastic income. J. Econom. Theory, 72:33-73, 1997.

[DFSZ97] D. Duffie, W. Fleming, M. Soner, and T. Zariphopoulou. Heding in incomplete markets with HARA utility. J. Econ. Dymaics and Control, 21:753-782, 1997.

[DM82] C. Dellacherie and P.A. Meyer. Probabilities and Potential B: Theory of martingales, volume 2. North-Holland Publishing Company, 1982.

[DS94] F. Delbaen and W. Schachermayer. A general version of the fundamental theorem of asset pricing. Math. Ann., 300:463-520, 1994.

[DS97] F. Delbaen and W. Schachermayer. The Banach space of workable contingent claims in arbitrage theory. Ann. Inst. H. Pincaré Statist. Probab., 33:113-144, 1997.

[DS98] F. Delbaen and W. Schachermayer. The fundamental theorem of asset pricing for unbounded stochastic processes. Math. Ann., 312:215-250, 1998.

[DZ93] D. Duffie and T. Zariphopoulou. Optimal investment with undiversifiable income risk. Math. Finance, 3:135-148, 1993.

[EKJP98] N. El Karoui and M. Jeanblanc-Picque. Optimization of consumption with labor income. Finance Stoch., 2:409-440, 1998.

[ET76] I. Ekeland and R. Temam. Convex Analysis and Variational Problems. North-Holland American Elsevier, 1976.

[FK97] H. Föllmer and D. Kramkov. Optional decompositions under constraints. Probab. Theory Relat. Fields, 109:1-25, 1997.

[HK04] J. Hugonnier and D. Kramkov. Optimal investment with random endowment in incomplete markets. Ann. Appl. Probab., 14:845-864, 2004.

[HKS05] J. Hugonnier, D. Kramkov, and W. Schachermayer. On utility-based pricing of contingent claims in incomplete markets. Math. Finance, 15:203-212, 2005.

[HP93] H. He and H. Pages. Labor income, borrowing constraints, and equilibrium asset prices. Econ. Theory, 3:663-696, 1993.

[JS03] J. Jacod and A. N. Shiryaev. Limit Theorems for Stochastic Processes. Springer, 2003. 
[Kar89] I. Karatzas. Optimization problems in the theory of continuous trading. SIAM J. Control Optim., 27(6):1221-1259, 1989.

[Kar13] C. Kardaras. On the closure in the Emery topology of semimartingale wealth-process sets. Ann. Appl. Probab., 23(4):1355-1376, 2013.

[KK07] I. Karatzas and K. Kardaras. The numéraire portfolio in semimartingale financial models. Finance Stoch., 11:447-493, 2007.

[KLPO14] I. Klein, E. Lépinette, and L. Perez-Ostafe. Asymptotic arbitrage with small transaction costs. Finance Stoch., 18(4):917-939, 2014.

[Kra96] D. O. Kramkov. Optional decomposition of supermartingales and hedging contingent claims in incomplete security markets. Probab. Theory Related Fields, 105:459-479, 1996.

[KŽ03] I. Karatzas and G. Žitković. Optimal consumption from investment and random endowment in incomplete semi-martingale markets. Ann. Probab., 31:1821-1858, 2003.

[Mos15] O. Mostovyi. Necessary and sufficient conditions in the problem of optimal investment with intermediate consumption. Finance Stoch., 19:135-159, 2015.

[Mos17] O. Mostovyi. Optimal investment with intermediate consumption and random endowment. Math. Finance, 27(1):96-114, 2017.

[MP01] M. Mnif and H. Pham. Stochastic optimization under constraints. Stochastic Process. Appl., 93:149-180, 2001.

[MT07] S. Malamud and E. Trubowitz. The structure of optimal consumption streams in general incomplete markets. Math. Finan. Econ., 1(2):129-161, 2007.

[Pro04] P. Protter. Stochastic Integration and Differential Equations. Springer, 2004.

[Rás18] M. Rásonyi. On utility maximization without passing by the dual problem. arXiv:1702.00982 [q-fin.PM], 2018.

[Rok10] D.B. Rokhlin. On the existence of an equivalent supermartingale density for a fork-convex family of stochastic processes. Mat. Zametki,, 87(4):594-603, 2010.

[Ž05] G. Žitković. Utility maximization with a stochastic clock and an unbounded random endowment. Ann. Appl. Probab., 15:748-777, 2005.

[XM16] H. Xing and A. Matoussi. Convex duality for Epstein-Zin stochastic differential utility. Math. Finance, 2016. to appear in.

[Yu15] X. Yu. Utility maximization with addictive consumption habit formation in incomplete semimartingale markets. Ann. Appl. Probab., 25(3):1383-1419, 2015.

Oleski Mostovyi, Department of Mathematics, University of Connecticut, Storrs, CT 06269, United States

E-mail address: oleksii.mostovyi@uconn.edu

Minai Sîrbu, Department of Mathematics, University of Texas at Austin, Austin, TX 78712, UNITED STATES

E-mail address: sirbu@math.utexas.edu 